\title{
Der Badearzt Asklepiades und seine bithynische Heimat*
}

Von Jost Benedum

Bithynien, das in der Nordwestecke Kleinasiens gelegene Land, hat im Verlauf der griechisch-römischen Antike eine Reihe namhafter Ärzte hervorgebracht, unter denen vor allem zwei die Entwicklung der Medizin entscheidend beeinflußt haben.

Der erste, Herophilos, war um 300 v. Chr. in Chalkedon ${ }^{1}$, einer der ältesten Küstenstädte des Landes, geboren. Er gilt als der Begründer einer systematischen Anatomie, die nicht zuletzt auch die Voraussetzung für jene Geburtshilfe bildete, die mit dem ebenfalls aus Bithynien stammenden Arzt Demetrios von Apameia $^{2}$ um 100 v. Chr. einen ersten Höhepunkt erlebte.

Der zweite, kaum weniger berühmte Bithynier war jener Arzt Asklepiades, der nicht nur ähnlich wie sein Landsmann Herophilos zahlreiche Anhänger fand und den Weg für eine der bedeutendsten Medizinschulen der Antike bereitete, sondern darüber hinaus erstmalig mit Erfolg griechische Medizin in Rom heimisch machte.

Über seine Person, die schon in der Antike mit der des gleichnamigen Rhetors Asklepiades von Myrleia ${ }^{3}$ verwechselt wurde, besitzen wir nur sehr lückenhafte und zudem widersprüchliche Zeugnisse. Denn einerseits tragen die Nachrichten deutlich sagenhafte Züge und entstammen einer Mythenbildung, die sich schon früh des Bithyniers bemächtigte und ihn als "Abgesandten des Himmels» und als «Totenerwecker» feierte ${ }^{4}$. Andererseits weisen die Testimonien eine vom Vorwurf der Zertrümmerung der Antiquitas bis zur Bezichtigung der Scharlatanerie reichende, oft hemmungslose Polemik auf, die aus dem Lager seiner Gegner rührt, unter denen Galen als der zwar sicher prominenteste, kaum aber objektivste Wortführer hervorragt ${ }^{5}$. Allein der Nichtarzt Celsus ${ }^{6}$, der Asklepiades einen « multarum rerum auctor bonus » nennt, stellt eine rühmliche Ausnahme dar, da er sich einer sine ira et studio konzipierten Kritik befleißigt. Ähnlich erblickt auch Scribonius Largus ${ }^{7}$ in ihm einen «maximus auctor medicinae», ohne jedoch gleichzeitig darauf zu verzichten, für Asklepiades Partei zu ergreifen und dessen verleumderischen Widersachern «Mord und Tempelschändung» vorzurücken.

Welchen von diesen Urteilen man sich auch immer anschließen mag, sicher

* Teile des Aufsatzes lagen einem Vortrag zugrunde, der am 18. September 1972 in Göttingen gehalten wurde. In seiner jetzigen Form ist er Teil der ungedruckten Festschrift für Gerhard Müller, zum 70. Geburtstag von Kollegen und Schülern dargebracht, Gießen 1977. 
dürfte sein, daß weder die Verteuflung durch seine Gegner noch die Vergottung durch seine Anhänger seiner Person voll gerecht werden. So kann man nicht übersehen, daß Epikur, einer der geistigen Väter des Bithyniers und wie dieser ein "Graius homo», zur selben Zeit von dem in Rom lebenden Dichter Lukrez ${ }^{8}$ als «göttlicher Himmelsstürmer» verherrlicht wurde, ohne deshalb den Ruf eines Komödianten oder Scharlatans zu verdienen. Auch kann gerade die Wiederbelebung eines Scheintoten - denn um eine solche handelt es sich bei der sogenannten Totenerweckung - kaum als Beweis für raffinierte Spekulation, Reklamesucht und fehlende sittliche Integrität gewertet werden. Dies legt schon Celsus ${ }^{9}$ nahe, der die Erweckungsgeschichte wohl am sachlichsten referiert und in diesem Zusammenhang Asklepiades ausdrücklich mit jenen «guten Ärzten» auf eine Stufe stellt, die zwischen den notae vitae finitae und den notae mortis futurae zu unterscheiden vermögen. Die sorgfältige Kenntnis der signa vitae bzw. mortis bescheinigt auch noch Apuleius ${ }^{10}$, der darüber hinaus anmerkt, Asklepiades sei durch genaue Inspektion und eingehende Palpation zu dem Ergebnis gelangt, daß der Totgeglaubte noch lebe. Zudem besitzt die Frage nach der Erkennung des Scheintodes eine gewisse Tradition ${ }^{11}$, vor deren Hintergrund gerade der Bithynier Asklepiades gesehen werden muß. Ohne hier ausführlicher darauf eingehen zu können, sei nur an den bithynischen Philosophen Herakleides Pontikos erinnert, mit dem Asklepiades nicht nur die Lehre von den «fugenlosen Körperchen», den Atomen, teilt. Denn seine Schrift «Über die Scheintote oder die Krankheiten », die bekanntlich die Erweckung der scheintoten Peisianax-Tochter Pantheia durch Empedokles und dessen Überlegenheit über die den wahren Sachverhalt verkennenden Ärzte schilderte, bemühte sich um das Verständnis des Scheintod-Problems als Voraussetzung für seine ärztliche Erkennung ${ }^{12}$. Ebenso setzt sich noch Galen ${ }^{13}$ an mehreren Stellen mit den physiologischen Grundlagen dieses Phänomens auseinander und zieht hierzu sogar den totenähnlichen Schlaf der Tiere heran. Dabei beruft sich der Pergamener jedesmal auf Herakleides Pontikos, übergeht aber bezeichnenderweise Asklepiades, der gerade in diesem Zusammenhang sicherlich nicht von der bissigen Kritik Galens verschont geblieben wäre, wenn seine «Totenerweckung» Anlaß dazu geboten hätte. Man wird daher zu Recht vermuten können, daß sich auch Asklepiades mit dieser Frage beschäftigt hat, zumal ihm die zitierte Schrift seines Landsmannes schwerlich unbekannt geblieben sein dürfte. In jedem Fall scheint sich hinter seiner «Totenerweckung» mehr als nur Komödiantentum zu verbergen, wenngleich ihre anekdotenhafte Ausschmückung ihm wie auch dem späteren großen arabischen Arzt Rhases ${ }^{14}$, den die Legende ebenfalls als «Totenerwecker» feierte, zu willkommenem Ruhm und Ansehen verhalf. 
Schließlich vermag die Beurteilung, die Herakleides Pontikos durch die Nachwelt erfahren hat, gerade wegen ihrer verblüffenden Ähnlichkeit mit der des Asklepiades zu zeigen, welchen Wahrheitsgehalt man insbesondere einzelnen verzerrenden Äußerungen beimessen darf. Im Falle des Herakleides Pontikos hat nämlich die Forschung inzwischen mit guten Gründen ein Bild entworfen, das von den negativen Urteilen seiner ärgsten Kritiker weitgehend befreit ist. Denn von den einen wegen seiner kühnen Gedanken und seiner unerhörten schriftstellerischen Begabung gerühmt, wurde er von den anderen wegen seiner Eitelkeit, Schwindelhaftigkeit und Scharlatanerie gerügt. Ein Orakelspruch soll ihm sogar die Heroisierung verheißen haben, doch traf ihn angeblich der Schlag, als ihm die Botschaft überbracht wurde. Auch hier wissen wir zwar im einzelnen nicht mit Bestimmtheit zu sagen, inwieweit Wahrheit und Persiflage miteinander verbunden sind. Doch galt Herakleides Pontikos wie sein bithynischer Landsmann Asklepiades lange Zeit sicher zu Unrecht als Scharlatan, wenngleich beiden Bithyniern abenteuerhafte Züge nicht abzusprechen sind.

Vor diesem Hintergrund miteinander rivalisierender und daher nur schwer zu vereinbarender Nachrichten versteht es sich, daß eine exakte Trennung von «Dichtung und Wahrheit» im Wirken des zu seiner Zeit zweifellos großen Arztes und damit eine gerechte Beurteilung der Persönlichkeit des Asklepiades bis heute nicht restlos gelungen ist. So ist denn auch trotz zahlreicher verdienstvoller Einzeluntersuchungen ${ }^{15}$, die sich mit Leben und Werk des Bithyniers auseinandersetzen, immer noch eine Reihe von Fragen ungelöst ${ }^{16}$. Auch stellt nicht zuletzt eine modernen Anforderungen genügende Fragmentsammlung ${ }^{17}$ nach wie vor ein Desiderat dar.

Einer dieser Fragen, nämlich der nach der Abstemplung des Asklepiades als «Kaltwasserdoktor» seit Varro ${ }^{18}$, ist der Verfasser vor Jahren am Beispiel der «Balnea pensilia» nachgegangen ${ }^{19}$. Gestützt auf literarische Zeugnisse konnte hierbei der «Warmwasserdoktor» in sein Recht gesetzt und zugleich deutlich gemacht werden, daß der auf Enantiose fußenden Solidarpathologie folgerichtig auch eine nach dem Prinzip «contraria contrariis» arbeitende Therapie zur Seite stand, die u. a. auch eine gleichwertige Anwendung von Kalt- und Warmbädern verlangte. Wenn in Ergänzung hierzu noch einmal der «Warmwasserdoktor» aufgegriffen wird, so deshalb, weil bislang von der Medizingeschichte nicht herangezogene epigraphische und numismatische Zeugnisse eine Erklärung auf die Frage zu geben vermögen, weshalb Asklepiades gerade den Warmbädern innerhalb seiner physikalisch-diätetischen Therapie eine so zentrale Stellung einräumte, daß er zusammen mit dem Ingenieur Sergius Orata ${ }^{20}$ sogar den Ausbau hypokaustierter Baderäume in Rom vorantrieb. Zwar weiß man seit langem, daß so- 
wohl Erasistratos ${ }^{21}$ - im Anschluß an seinen Lehrer Chrysipp - durch seine hydrotherapeutischen Verordnungen wie auch Kleophantos ${ }^{22}$ durch seine Weinbehandlung den «Wasser- bzw. Weindoktor» Asklepiades stark beeinflußt haben ${ }^{23}$. Dennoch weist schon Meyer-Steineg zu Recht auf die auffallend große Bedeutung der Wasseranwendung bei Asklepiades hin, ohne freilich eine hinlängliche Erklärung für diese Neuerung geben zu können, die dem Graeculus in Rom so wesentlich zum Erfolg verhalf. Mit Plinius erblickt er zwar einen der Hauptgründe hierfür in dem Bestreben des Bithyniers, «den Neigungen seiner Zeit entgegenzukommen ». ${ }^{24}$ Doch dürfte mit dieser Berufung auf den Zeitgeist kaum die Frage nach dem Anteil des Asklepiades beantwortet sein. Denn man wird davon auszugehen haben, daß der Bithynier schon vor seiner Ankunft in Rom praktische Erfahrungen als Badearzt besessen hat, ebenso wie er sich auch seine theoretischen Kenntnisse in atomistischer Philosophie - in Anlehnung an seinen Landsmann Herakleides - nicht erst in der neuen Weltstadt angeeignet haben dürfte ${ }^{25}$. Damit soll natürlich nicht in Abrede gestellt werden, daß Asklepiades' mechanistische Vorstellungen von Ätiologie und Therapie der Krankheiten in Rom nicht auf besonders fruchtbaren Boden fielen, nicht zuletzt weil sich der Atomismus epikureisch-lukrezischer Prägung hier inzwischen zunehmender Beliebtheit erfreute. Insgesamt gesehen dürfte sogar seine Spekulationen abholde «Methode» dem nüchtern denkenden Römer weitgehend entgegengekommen sein. Auf die offene Frage, wo Asklepiades die Impulse empfangen hat, die ihn zum «Wasserheilarzt» schlechthin werden ließen, vermag ein Blick auf seine bithynische Heimat und seinen Ausbildungsgang nähere Auskunft zu geben. Dabei sei ausdrücklich hervorgehoben, daß die Kennzeichnung des Asklepiades als Hydrotherapeut nicht eine neuerliche einseitige Abstemplung bedeutet, sondern lediglich einen Aspekt seiner bekanntlich vielseitigen ärztlichen Tätigkeit hervorhebt.

Asklepiades stammte aus Bithynien, das, wie Strabon ${ }^{26}$ betont, eine Reihe bemerkenswerter Männer hervorgebracht hat. Während also sein Heimatland feststeht ${ }^{27}$, herrscht über seine Geburtsstadt schon seit der Antike Uneinigkeit. So schreibt noch H. Haeser ${ }^{28}$, es sei dunkel, welches Prusa die Vaterstadt des Asklepiades gewesen sei, und diese Aporie besteht denn auch bis heute, obwohl sich die moderne medizinhistorische Forschung ${ }^{29}$ - freilich ohne Angabe von Gründen - inzwischen fast einstimmig für den «Prusaner» entschieden hat.

Verantwortlich für diese Unsicherheit ist zunächst der Umstand, daß das Altertum drei Städte in Bithynien kannte, die wegen ihrer ähnlich lautenden Namen trotz L. Roberts ${ }^{30}$ mehrfach eindringlich erhobener Appelle bis in die Ge-

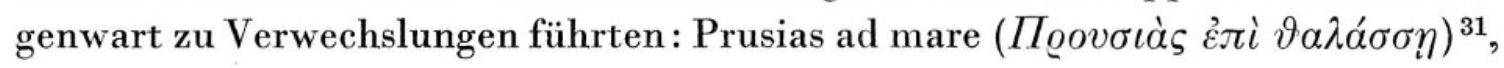




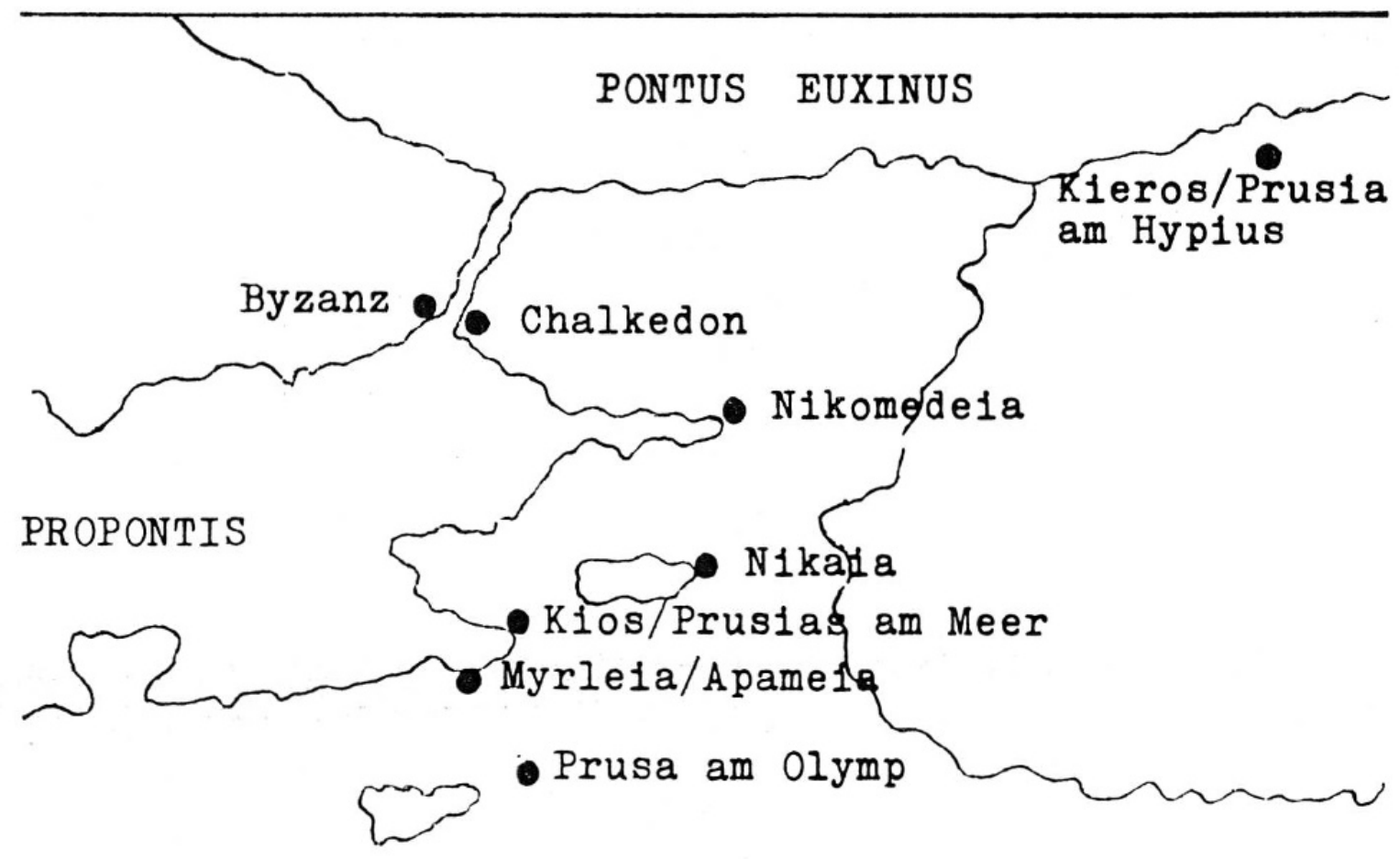

Abb. 1. Kartenskizze

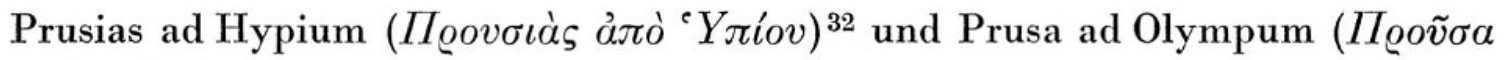
$\pi \varrho o ̀ \varsigma ~ ' O \lambda v ́ \mu \tau \omega)^{33}$. Die älteste dieser Städte, «Prusias am Meer», hat darüber hinaus im Verlauf ihrer Geschichte mehrmals den Namen geändert: Ursprünglich von milesischen Siedlern um 625 v. Chr. gegründet, hieß sie bis zu ihrer vollständigen Zerstörung im Jahre 202 v. Chr. Kios, sodann nach ihrem Wiederaufbau durch Prusias I etwa 200 Jahre lang "Prusias am Meer», um in claudischer Zeit wieder ihren alten Namen Kios (Cius) anzunehmen ${ }^{34}$. Ähnlich erging es «Prusias am Hypius», das seinen alten griechischen Pflanznamen Kieros ebenfalls unter Prusias I eintauschen mußte. Allein «Prusa am Olymp» scheint seinen Königsnamen als junge Gründung unverändert beibehalten zu haben, wobei freilich seine Vorgeschichte bis heute stark umstritten ist.

Vor diesem Hintergrund irreführender Städtenamen und Umbenennungen ist es zunächst verständlich, da $\beta$ eine exakte Bestimmung des Geburtsortes des Asklepiades nur schwer gelingen kann $^{35}$, zumal schon die antike literarische Überlieferung gerade hinsichtlich der Heimatstadt des Bithyniers eine große Unsicherheit der Schreibweise verrät. So bieten beispielsweise die Handschriften der

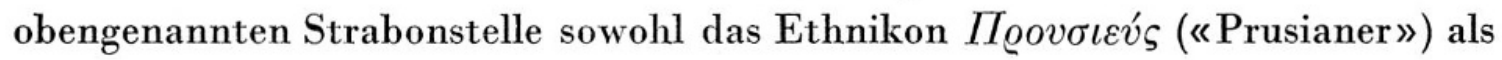




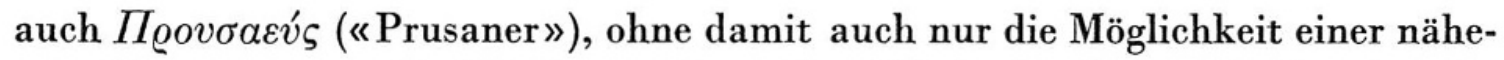
ren Abgrenzung an die Hand zu geben. Erst Plinius ${ }^{36}$ scheint mit der Angabe «Asclepiadi Prusiensi» die Wahl zwischen «Prusias am Meer» und «Prusias am Hypius» nahezulegen, und wenn man der Überlieferung der pseudo-galenischen Introductio ${ }^{37}$ Glauben schenken will, stammte Asklepiades in der Tat aus «Prusias am Meer». Denn dort heißt es am Ende einer Aufzählung, die mit Hippokrates beginnt und mit Asklepiades endet, von letzterem: «Asklepiades der Bithynier, der Kianer, der auch der Prusianer genannt wurde.»

Welche textkritischen Bedenken ${ }^{38}$ man auch immer gegen diese Stelle hegen mag, der Verfasser der Introductio hat eine selten klare Ortsangabe gemacht, der keines der bislang bekannten Testimonien widerstreiten kann. Im übrigen dürfte diese Stelle gleichzeitig einen wertvollen Anhaltspunkt für die fragliche Abfassungszeit der pseudo-galenischen Introductio bieten. Denn nach Ausweis der Münzen ${ }^{39}$ erhielt Prusias ad mare seinen ehemaligen Namen Kios (Cius) offiziell erst in claudischer Zeit wieder, so daß in der Kennzeichnung des Asklepiades als Kianer ein terminus post quem für diese eisagogische Schrift erblickt werden $\mathrm{kann}^{40}$. Darüber hinaus wäre es nicht verwunderlich, wenn sich unter den Vor-

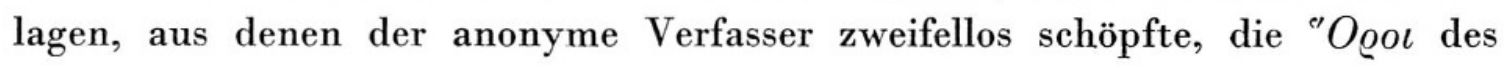
Asklepiades befunden hätten. Aus ihnen könnte nämlich sehr wohl die exakte geographische Angabe des Geburtsortes unseres Arztes stammen ${ }^{41}$.

Liegt damit aufgrund der Überlieferung der pseudo-galenischen Introductio «Prusias am Meer» als Geburtsort des Asklepiades fest ${ }^{42}$, so schließt dies natürlich nicht aus, daß sich der "Prusianer» auch zeitweilig in «Prusa am Olymp » aufgehalten hat, zumal beide Städte in unmittelbarer Nachbarschaft gelegen sind ${ }^{43}$. Für einen Aufenthalt auch in «Prusa am Olymp» spricht zunächst die Tatsache, daß der Bithynier in mehreren Städten seiner Heimat praktiziert hat. So verbrachte er eine gewisse Zeit am Hellespont, insbesondere in Parium. Denn in einem bei Oribasius ${ }^{44}$ erhaltenen Fragment berichtet Asklepiades von zwei Fällen einer spontanen Hüftgelenksluxation, deren eine er in Parium beobachtet hat. Ebenso überliefert Caelius Aurelianus ${ }^{45}$, daß Asklepiades bei der Pleuritis die Anwendung der Venaesectio von der Landschaft, in der die Krankheit zur Behandlung kommt, abhängig gemacht habe. Er habe nämlich festgestellt, daß der Aderlaß in Parium und am Hellespont nütze, in Athen und Rom dagegen oft schade.

Stellen diese beiden Zeugnisse sicher, daß Asklepiades schon vor seinem RomAufenthalt wenigstens in Parium und anderen Städten des Hellesponts als Arzt tätig war, so dürfte auch ein Aufenthalt in Prusa am Olymp nicht von vornherein auszuschließen sein. Freilich kann hieraus nicht abgeleitet werden, Asklepiades 
sei in Prusa geboren. Wir kennen zwar einen C. Calpurnius Asclepiades - er wurde am 5. März $87 \mathrm{n}$. Chr. in Prusa am Olymp geboren und verstarb im Jahre 157 n. Chr. in Capena -, der als Arzt in Rom unter Traian tätig war und sogar vom Kaiser für seine Eltern, sich und seine vier Brüder das römische Bürgerrecht erlangte ${ }^{46}$. Doch wissen wir nicht, ob er zu den Nachfahren des großen Asklepiades zählte, wenngleich er mit einiger Sicherheit der methodischen Schule zuzurechnen sein dürfte. Selbst der Hinweis, daß der Tierarzt Apsyrtus ${ }^{47}$ ebenfalls aus Prusa stammte und dazu noch ein Buch seiner Hippiatrika einem Mitbürger namens Asklepiades zueignete, den er als den «größten Arzt» bezeichnet, kann die Stadt als Geburtsort unseres Asklepiades kaum wahrscheinlicher machen. Viel eher ist denkbar, daß Asklepiades als Bürger von «Prusias am Meer»

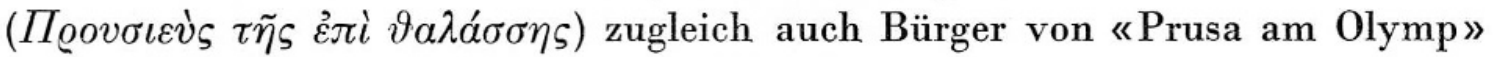

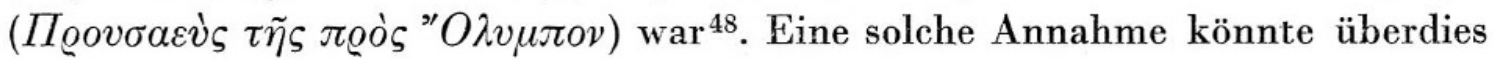
die Unsicherheit schon der antiken Autoren bei der Frage nach dem Geburtsort nachträglich erklären.

Aber nicht nur die im einzelnen unbekannten Stationen des Bithyniers lassen vermuten, daß der «Wasserdoktor» sich auch in Prusa vorübergehend aufgehalten hat, das immerhin schon im 1.Jahrhundert n. Chr. eine Bibliothek ${ }^{49}$ besaß, wenngleich es nach dem Zeugnis eines seiner berühmtesten Söhne, des Dio

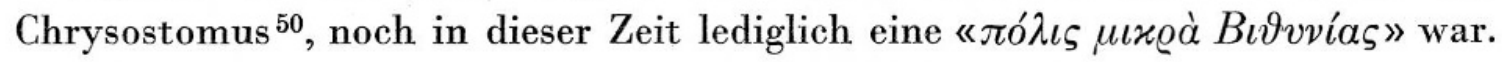
Den wichtigsten Hinweis hierfür gibt vielmehr eine Münze ${ }^{51}$ aus «Prusa am Olymp», deren Aussage gerade den "Warmwasserdoktor» in einem charakteristischen Licht erscheinen läßt. Abgebildet ist auf ihrer Vorderseite der Kopf der Kaiserin Julia Mamaea, der Mutter des Alexander Severus. Entstammt dieses Zeugnis damit zwar erst der Zeit zwischen $222-235 \mathrm{n}$. Chr. ${ }^{52}$, so ist dennoch die Darstellung auf der Rückseite von besonderem Interesse. Wiedergegeben ist hier die tetrastyle Front eines Gebäudes, dessen Innenraum von einem auf Säulen ruhenden Tonnengewölbe überspannt wird ${ }^{53}$. Vor diesem Kuppelbau lagern einander gegenüber zwei weibliche Gestalten, die beide mit der einen Hand je ein Gefäß vor ihren Knien halten, dem Wasser entströmt. In der anderen Hand tragen sie jeweils ein Schilfrohr. Es kann nun aufgrund der Attribute kein Zweifel darüber bestehen, daß es sich bei den Dargestellten um Quellnymphen ${ }^{54}$ handelt, die, wie L. Robert überzeugend erschloß, hier vor einem Quellhaus liegen, das zu einem größeren Thermalbezirk gehörte und dessen Fertigstellung durch das Münzbild gefeiert wird.

Sucht man nach einer Bestätigung für diese Münznachricht, so bietet sich zunächst eine Inschrift ${ }^{55}$ aus Athen an, deren Schriftcharakter ebenfalls in die Zeit um $200 \mathrm{n}$. Chr. weist. Die in Distichen abgefaßte Grabschrift nennt einen vermut- 

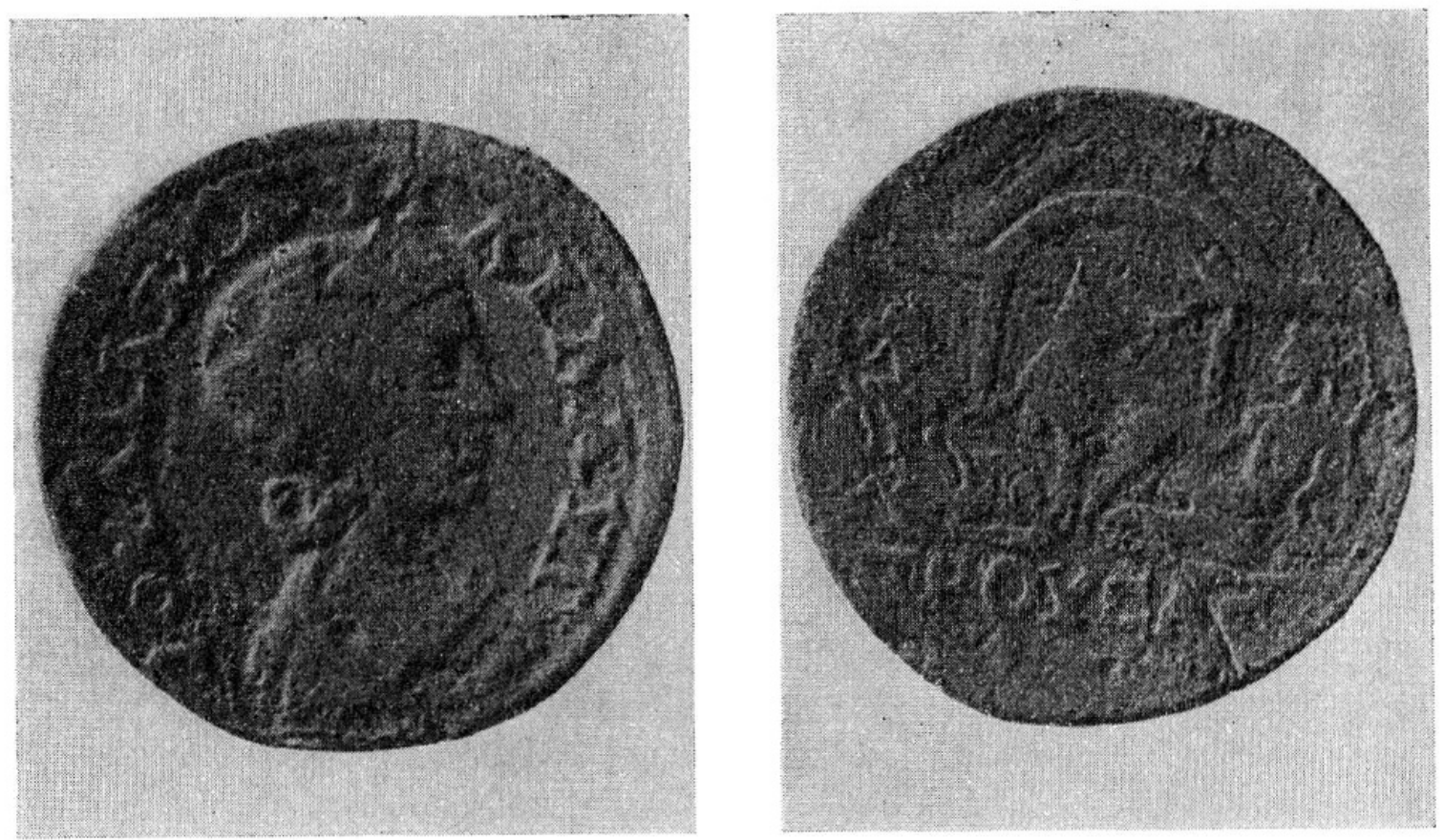

Abb. 2. Münze aus Prusa am Olymp (zw. 222-235 n. Chr.).

Vs.: Kopf der Julia Mamaea rechtshin. Aufschrift: IOY $\triangle I A$ MAMAIA AYT

Rs.: Tetrastyle Gebäudefront mit zwei Quellnymphen. Aufschrift: $\Pi P O Y \Sigma A E[\Omega N]$

Aufnahme aus: E. Babelon/Th. Reinach, Recueil des monnaies grecques, Bd. 1, Fasz. 4, Paris 1912, Tafel CII, 10

Abb. 3. Grabepigramm aus Athen (um 200 n. Chr.). Aufnahme aus: W. Peek, Griechische Epigramme III. Mitt. d. Dt. Arch. Inst. Athen 66 (1941), Tafel 36,3

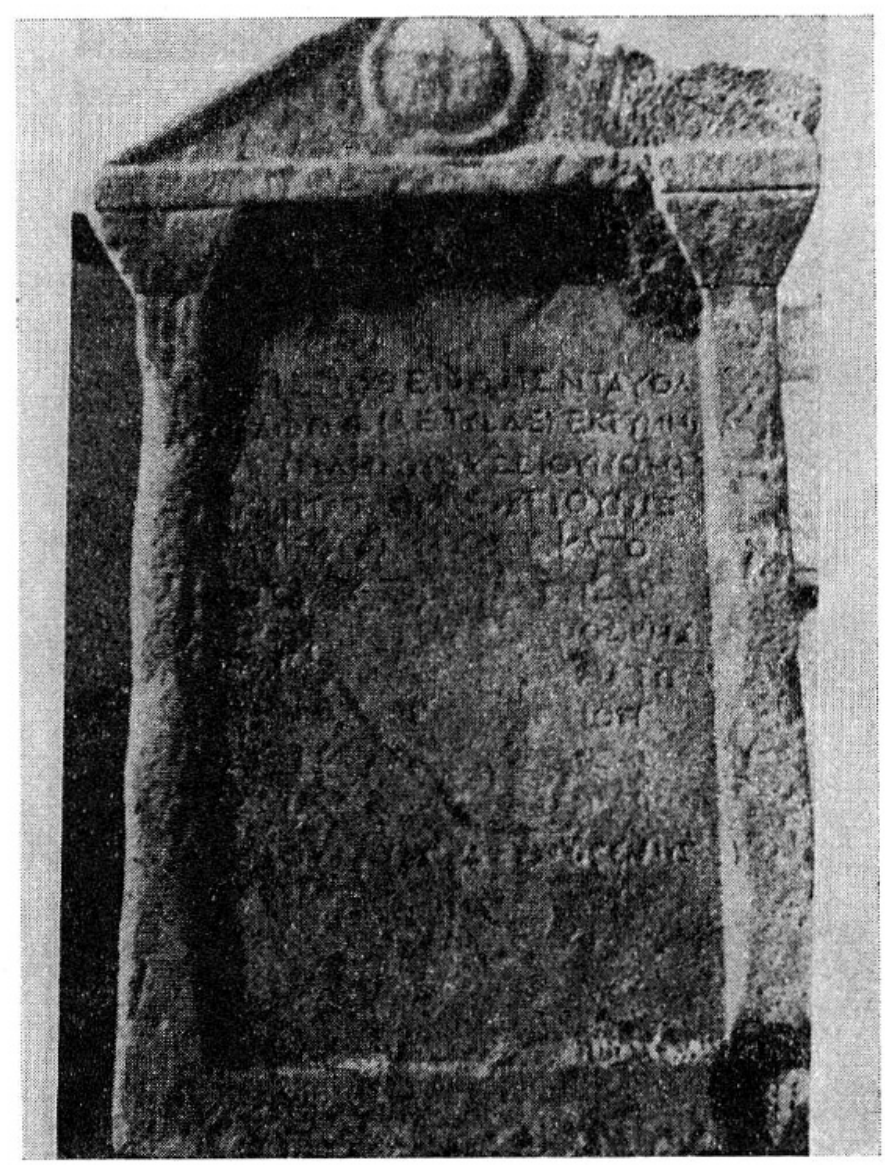


lich in jungen Jahren verstorbenen Hermogenes, der aus jenem Prusa stammte, «das aus seiner Erde die warmen Bäder der Nymphen hervorströmen läßt». ${ }^{56}$ Zieht man hierzu außerdem das Zeugnis bei Galen ${ }^{57}$ heran, wonach Prusa am Olymp eine deshalb nicht gerade alltägliche Thermalquelle besitzt, weil sie nicht nur weniger als zehn Stadien ( = rund $2 \mathrm{~km}$ ) von der Stadt entfernt liegt, sondern zugleich auch von unterschiedlichen Quellen gespeist wird und daher ein verschieden heilkräftiges Wasser führt, so dürfte die Deutung des Münzbildes hinlänglich bestätigt sein. Denn vermutlich spielte Galen damit auf die Quellen des Pieros und des Odryses an, die schon F. Imhoof-Blumer ${ }^{58}$ in den beiden Nymphen des Münzbildes personifiziert sah. Aber auch Athenaeus ${ }^{59}$, dessen «Tischgespräche» nicht vor $192 \mathrm{n}$. Chr. erschienen sind, kennt die natürlichen Thermalquellen von Prusa und hebt sie ausdrücklich als die «sogenannten Königlichen

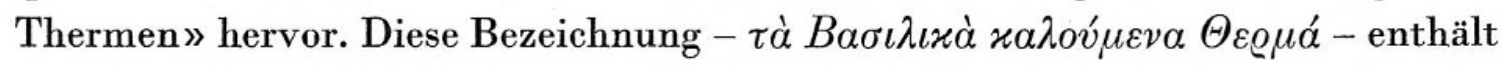
zugleich den wichtigen Hinweis, daß die warmen Heilquellen schon zur Zeit der bithynischen Könige und damit schon vor dem 1. Jahrhundert v. Chr. bestanden haben dürften. Denn Bithynien fiel nach dem Testament seines letzten Königs Nikomedes Philopator im Jahre 74 v. Chr. als Erbe den Römern zu, die das Land ab diesem Zeitpunkt als römische Kolonie einrichteten. Wenngleich wir Näheres über das Badeleben in Prusa am Olymp vor diesem Datum nicht erfahren, so wird man doch die Existenz dieser heilkräftigen Warmquellen gerade schon zur Zeit des «Warmwasserdoktors» Asklepiades voraussetzen dürfen.

Freilich scheinen die Thermalanlagen von Prusa auch Zeiten des Verfalls erlebt zu haben. Dafür spricht nicht zuletzt auch die Nachricht, daß Plinius der Jüngere, der zwischen 111 und 113 n. Chr. kaiserlicher Legat in Bithynien war, bei seiner Ankunft in Prusa am Olymp den Zustand des Badebezirkes so verwahrlost vorfand, da $\beta$ er den Kaiser Traian brieflich ${ }^{60}$ um die Erlaubnis bat, einen Wiederaufbau zu gestatten. In die Zeit kurz nach der Jahrhundertwende fallen auch die Bemühungen des Dio Chrysostomus, durch Abreißen baufälliger Gebäude, Errichtung einer Stoa und Bau von Wasserleitungen seiner Geburtsstadt ein neues Gesicht zu verleihen ${ }^{61}$. Wie Plinius ${ }^{62}$ berichtet, hatte Dio damals die Bauleitung übernommen. Was die «Königlichen Thermen» betrifft, so hat schließlich auch Kaiser Hadrian in einem Brief, den L. Robert ${ }^{63}$ auf einem Steinzeugnis von Prusa am Olymp wiedererkennen konnte, die $\vartheta \varepsilon \varrho \mu \grave{\alpha} z \delta \alpha \tau \alpha$ aufgegriffen. Leider ist jedoch der Brief derart zerstört, daß der Inhalt des Beschlusses über die Thermalquellen von Prusa nicht mehr rekonstruierbar ist.

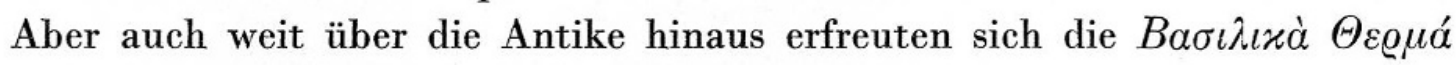
von Prusa großer Beliebtheit, nicht zuletzt wegen der Gunst, die ihnen die byzantinischen Kaiser entgegenbrachten. So hat schon L. Robert ${ }^{64}$ auf die Be- 
suche Konstantins VI. und seiner Mutter Irene im letzten Jahrzehnt des 8. Jahrhunderts (796) und Kaiser Leos VI. im 9. Jahrhundert hingewiesen. Sie alle begaben sich nach Prusa am Olymp, um dort zu «thermen». Besonders aufschlußreich ist in diesem Zusammenhang ein Epigramm des Leontius Scholasticus ${ }^{65}$

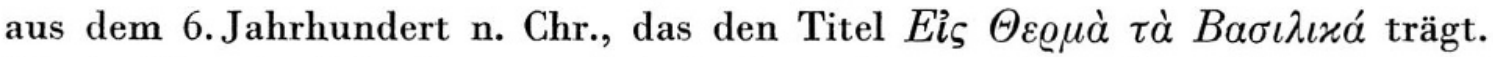
Hierin führt der Schreiber aus, daß die Bezeichnung $\Theta \varepsilon \varrho \mu \alpha \dot{\alpha} B \alpha \sigma \iota \lambda \eta_{i} \alpha$ schon auf die Alten zurückgehe. Denn sie hätten am meisten bestaunt, daß das Quellwasser künstlich weder erwärmt noch abgekühlt zu werden brauchte, da es gerade die richtige Temperatur besitze ${ }^{66}$. Dieser Hinweis auf die vorzügliche Temperatur des Thermalwassers von Prusa zielt dabei fraglos auf die ebenfalls in Bithynien am Eingang des Nikomedischen Golfes bei Yalova gelegenen «Pythischen Thermen ", ${ }^{67}$ deren Quellen jedoch so heiß waren, daß sie erst mit kaltem Wasser gekühlt werden mußten. So verwundert es auch nicht, daß die «Königlichen Thermen» von Prusa und die "Pythischen Thermen» von Yalova lange Zeit untereinander konkurrierten, zumal die Kaiserin Theodora (gest. 548 n. Chr.) sich zu den unweit von Konstantinopel gelegenen «Pythischen Thermen» zu begeben pflegte und ihr Gemahl Justinian dort sogar einen Palast hatte erbauen lassen ${ }^{68}$. Diese Rivalität zwischen beiden Badeorten findet denn auch in einem weiteren Epigramm ${ }^{69}$ unbekannter Zeit ihren Ausdruck, in dem der Verfasser jedoch den «Königlichen Thermen» von Prusa am Olymp den ersten Platz zuweist. Schließlich soll noch Süleyman der Prächtige ${ }^{70}$ (1520-66) in den Bädern von Prusa die Heilung seines Gelenkleidens gefunden haben.

Der osmanischen Zeit entstammen auch die wichtigsten Badeanlagen im modernen türkischen Bursa, die von zahlreichen Reisenden früherer Jahrhunderte gerühmt wurden ${ }^{71}$ und noch vom Touristen unserer Tage besucht werden. Unter ihnen sind in erster Linie das vom Schwiegersohn Süleymans des Prächtigen, dem Großwesir Rüstem Pascha, im Jahre 1526 erbaute Yeni Kaplica-Bad und das bereits um 1389 durch Sultan Murad I. errichtete Eski Kaplica-Bad zu nennen ${ }^{72}$. Wenngleich die Architektur dieser Anlagen die relativ späte Entstehung bestätigt, weisen doch einzelne Baustilelemente deutlich bis in byzantinische Zeit zurück. Auch läßt die am Grundriß abzulesende Einteilung in Apodyterium, Tepidarium und Calidarium die lange bis in die Antike zurückreichende Tradition erkennen ${ }^{73}$. Gerade das zuletzt genannte Eski Kaplıca-Bad, dessen Name «Alte, überdachte Heißquelle ${ }^{\mathbf{7 4}}$ bedeutet, verdient besondere Beachtung. Denn es liegt etwa $2 \mathrm{~km}$ nordwestlich von Bursa im jetzigen Stadtteil Çekirge und wird noch heute von heilkräftigen Warmquellen gespeist ${ }^{75}$, so daß nicht nur Galens Angabe, die heiBen Quellen entsprängen 10 Stadien von Prusa entfernt, eine Identifikation des Eski Kaplıca-Bades mit den antiken «Königlichen Thermen» nahelegt, sondern 


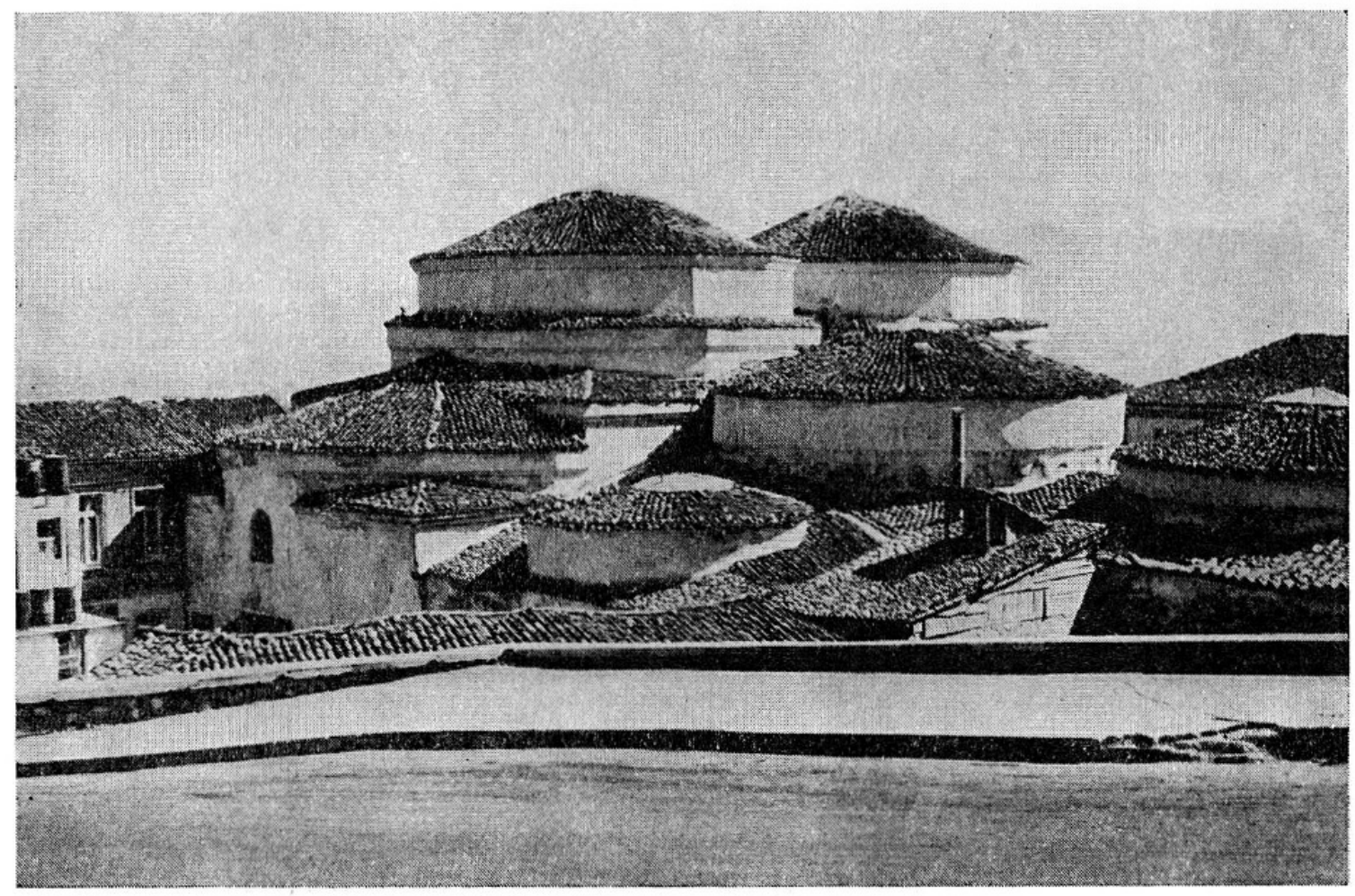

Abb. 4. Das moderne Eski Kaplica-Bad in Prusa am Olymp. Aufnahme aus: K. Eller/ D. Wolf, Das goldene Buch der Türkei, München 1964, Abb. 70

auch in dem beschriebenen Münzbild mit Badehaus und Quellnymphen geradezu die antike Darstellung eines Teiles dieses modernen Badebezirkes erblickt werden darf. Darüber hinaus weist das Quellwasser des Eski Kaplıca-Bades nach Messungen aus dem Jahre 1923 mit 42 bis $34^{\circ} \mathrm{C}$ im Becken ${ }^{76}$ genau jene Wärmegrade

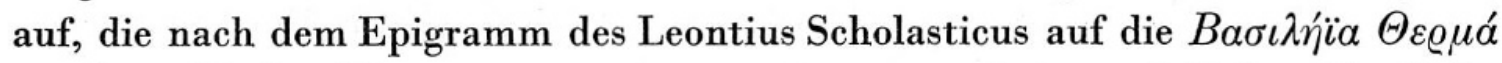
der menschlichen Körpertemperatur so weit entgegenkamen, daß eine künstliche Senkung der Wassertemperatur zum Erstaunen der Alten nicht erst erforderlich

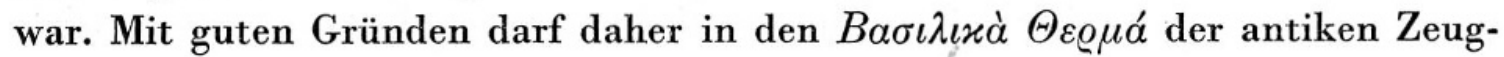
nisse das heutige Eski Kaplica-Bad wiedererkannt werden. Diese Identifikation setzt auch F. K. Dörner voraus, wenn er die $B a \sigma \iota \lambda \iota x \dot{\alpha} \Theta \varepsilon \varrho \mu \alpha ́$ im modernen Stadtteil Çekirge lokalisiert ${ }^{77}$.

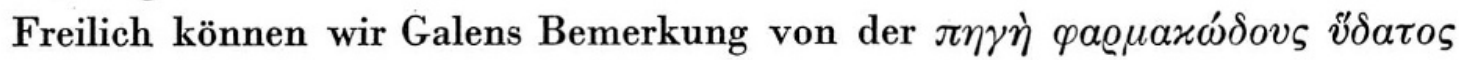
nicht genauer fassen, da antike Angaben über die spezifischen Bestandteile des Quellwassers fehlen. Moderne Analysen, die im 20. Jahrhundert unter anderem

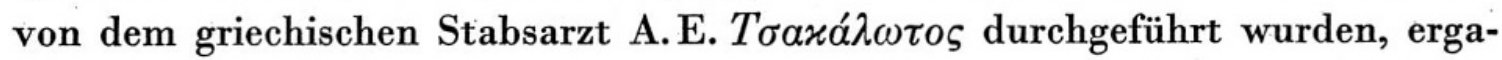
ben jedoch trotz der zum Teil starken Abweichungen der Meßwerte, daß das Thermalwasser unter anderem einen hohen Gehalt an Kalzium aufweist ${ }^{78}$. 

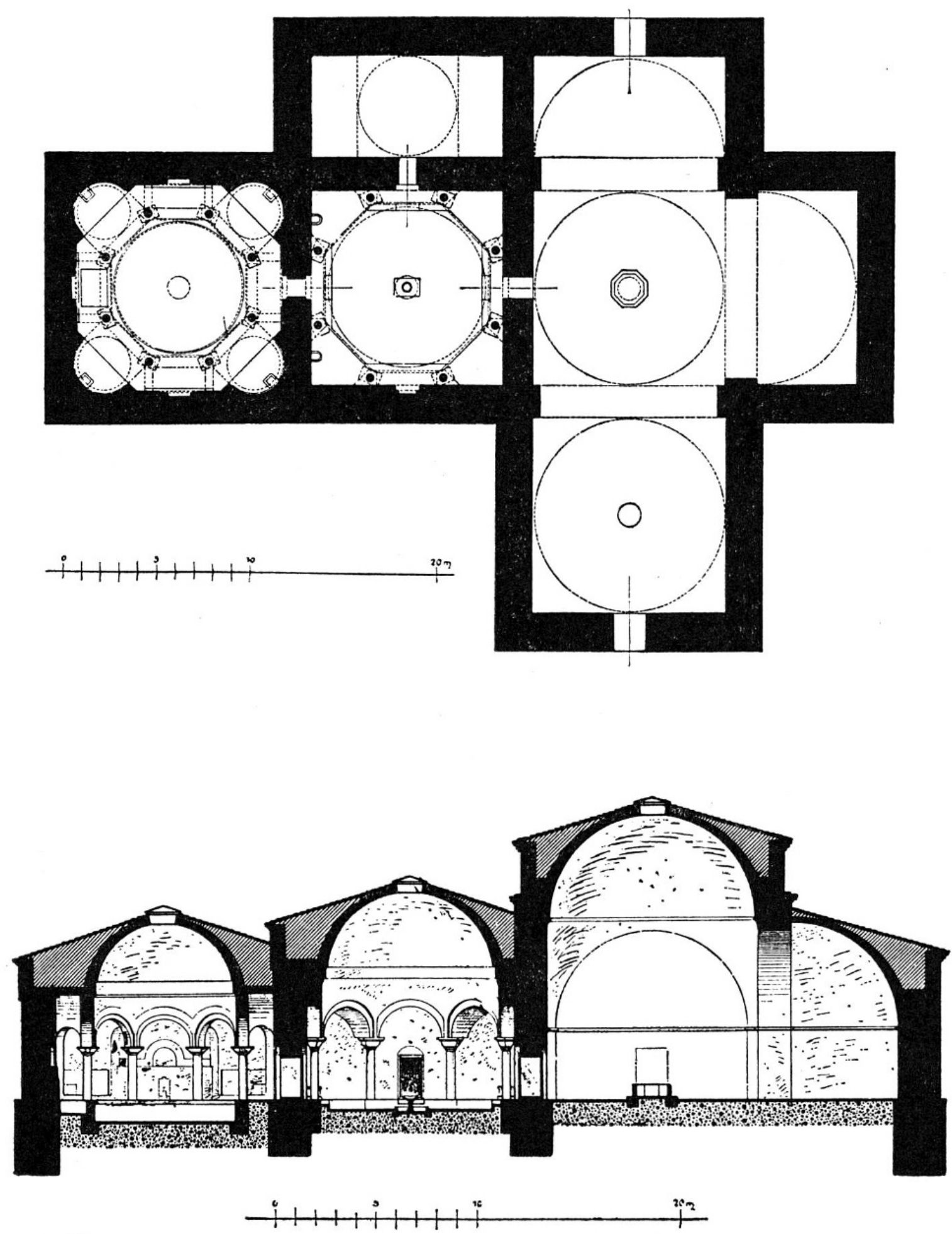

Abb. 5. Grundriß und Längsschnitt des Eski Kaplıca-Bades in Prusa am Olymp mit Apodyterium (rechts), Tepidarium (Mitte) und Calidarium (links). Aufnahme aus: H. Wilde, Brussa (Beiträge zur Bauwissenschaft, Heft 13), Berlin 1909, Abb. 117/118 


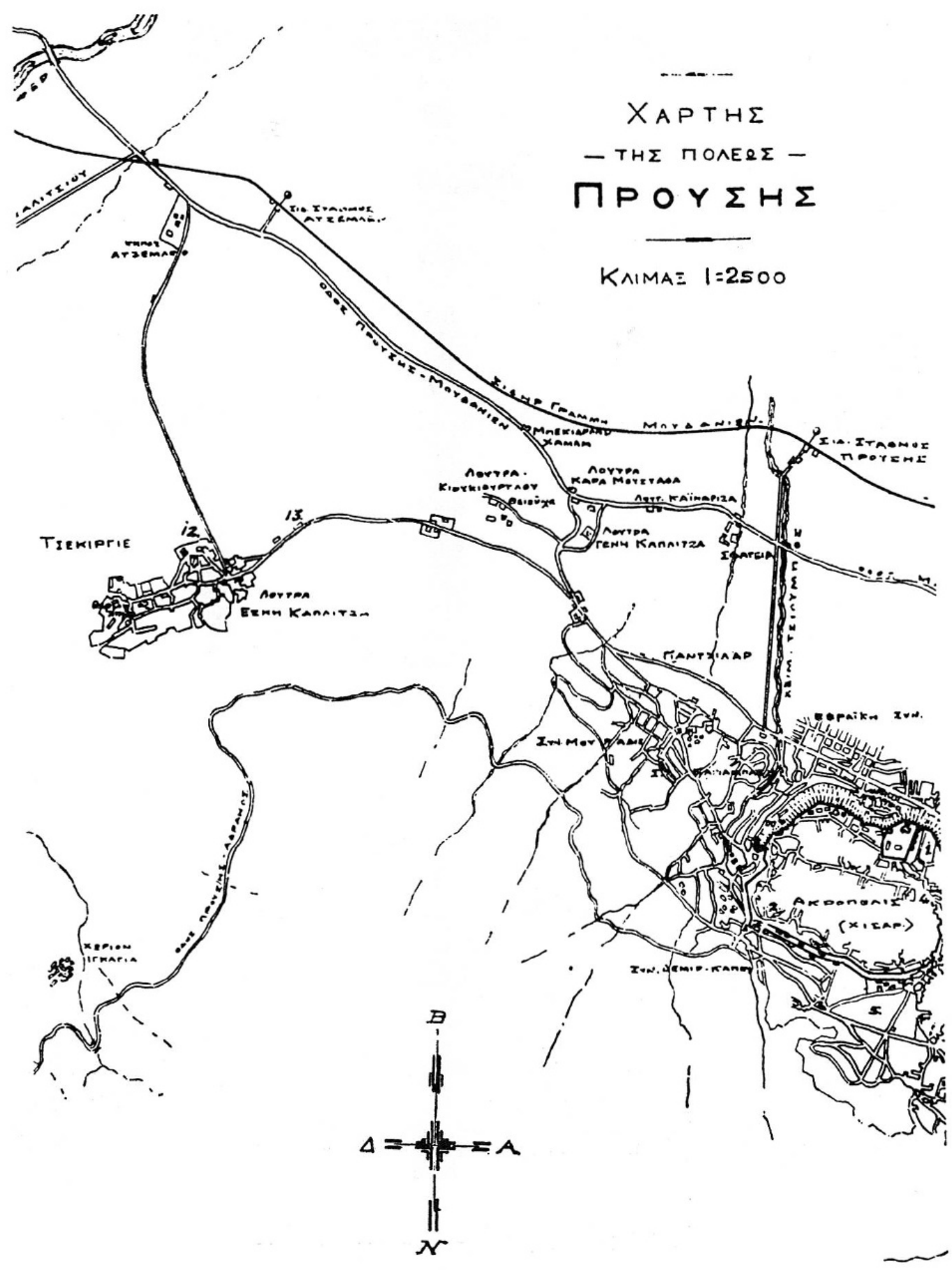

Abb. 6. Lageplan der Bäder von Prusa am Olymp mit dem Eski Kaplıca-Bad im Stadt-

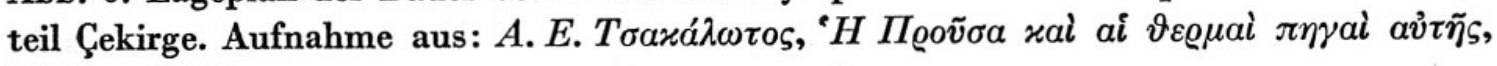

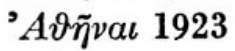


Nach alldem dürfte feststehen, daß die warmen Quellen von Prusa auf eine lange, von der Antike bis in die Moderne reichende Tradition zurückblicken. Daher kann auch der Behauptung von A.D.Mordtmann ${ }^{79}$, die Thermalquellen hätten im Altertum noch nicht existiert, nicht länger beigepflichtet werden. Vielmehr ist es wahrscheinlich, daß gerade die natürlichen Warmquellen schon in früher Zeit den Anlaß zur Gründung von Prusa am Olymp gegeben haben ${ }^{80}$. Wenigstens war Prusa nicht zuletzt auch wegen seiner heilkräftigen Quellen lange Zeit (1236-1453) Residenzstadt der osmanischen Sultane, welche die Stadt mit zahlreichen prachtvollen Bauwerken ausschmückten. So schreibt noch Helmuth von Moltke ${ }^{81}$ im Jahre 1836 über das «Grüne Bursa» ("Yesil Bursa»), wie es noch heute gerne genannt wird:

«Die ganze reich bebaute Gegend erinnert sehr an die Lombardei. Es ist in der That schwer zu entscheiden, welche der beiden Hauptstädte der osmanischen Herrscher die schönere Lage hat, die älteste oder die neueste, Brussa oder Konstantinopel. Nirgend habe ich eine weite, so durchaus grüne Landschaft gesehen. Überraschend ist der Wasserreichtum; überall rauscht ein Bach; mächtige Quellen stürzen sich aus dem Gestein, eiskalte neben dampfenden, und in der ganzen Stadt, in den Moscheen selbst sprudelt das Wasser aus zahlreichen Springbrunnen hervor.

Von der Annehmlichkeit der türkischen Bäder habe ich schon früher geschrieben. Die von Brussa zeichnen sich dadurch aus, daß sie nicht durch Kunst, sondern von Natur dergestalt geheizt sind, daß man es anfänglich für unmöglich hält, in das große klare Bassin zu steigen, ohne gesotten wieder herauszukommen.»

Die Untersuchung war ausgegangen von der Frage, wo Asklepiades aus Prusias am Meer jene Impulse empfangen haben mag, die ihn veranlaßten, den Kalt- und Warmbädern innerhalb seiner physikalisch-diätetischen Therapie eine so zentrale Stellung einzuräumen, daß noch Caelius Aurelianus rund fünf Jahrhunderte später ihn mit den Worten charakterisieren konnte: "Hic est ille Asclepiades frigidans atque calefacians.» Die Antwort lautet: In den berühmten Badeorten seiner bithynischen Heimat, unter denen neben Yalova am Nikomedischen Golf besonders Prusa am Olymp mit seinen «Königlichen Thermen» hervorragt.

Auf eine weitere Stadt sei abschließend nur hingewiesen. In Smyrna, dem Sitz der von Hikesios begründeten Erasistrateer-Schule, sind vom Spaten der Archäologen umfangreiche Badeanlagen freigelegt worden, zu denen auch das von heilkräftigen Warmquellen gespeiste sogenannte Agamemnon-Bad gehört. Zieht

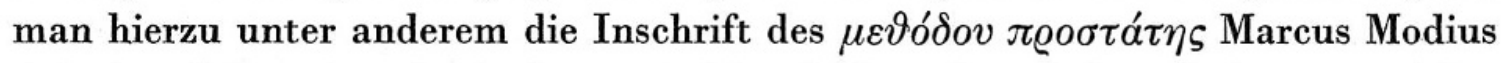
Asiaticus ${ }^{82}$ hinzu, so dürfte kaum ein Zweifel bestehen, daß auch Smyrna zu den Stationen des großen Bithyniers zu rechnen ist. Denn mit dieser Stadt ist nicht nur der Ort gewonnen, wo Asklepiades mit der erasistrateischen Schule in Berührung gekommen ist, mit der ihn ja trotz grundsätzlicher Unterschiede so 
manche für sein System charakteristische Lehre verbindet. Die angeführte smyrnäische Arztinschrift scheint vielmehr auch die Nachricht Strabons ${ }^{83}$ zu bestätigen, daß die dortige Schule der Erasistrateer bereits um 50 v. Chr. unter den Einfluß der von Asklepiades vorbereiteten methodischen Lehre geraten war ${ }^{84}$.

Man wird daher am Ende festhalten dürfen, daß beide Orte, Smyrna und Prusa am Olymp, mit ihren ausgedehnten Thermalanlagen zu jenen Stationen zu rechnen sind, auf denen der junge Bithynier Asklepiades aus Prusias am Meer bereits vor Aufbruch in die Weltstadt Rom die Anregungen empfangen hat, die ihn zum Wasserarzt schlechthin werden ließen. Der einseitigen Abstemplung als «Psychrolutes» widerstreiten sowohl die literarischen wie auch die epigraphischnumismatischen Zeugnisse.

\section{Anmerkungen}

1 Chalkedon als Geburtsort des Herophilos ist durch Pseudo-Galen, Introductio cap. 4 (XIV,

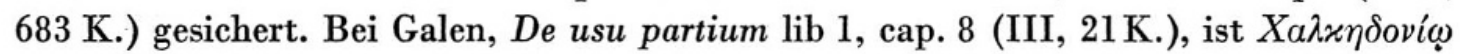

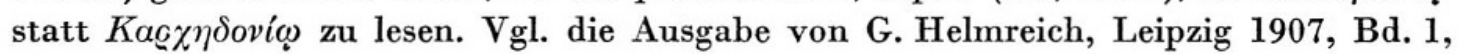
S. 15.

${ }^{2} \mathrm{Da}$ es sich hierbei um das bithynische und nicht um das syrische Apameia handelt, wo z.B. Archigenes geboren wurde, ist mehrfach bezeugt. Vgl. z. B. Galen, De compos. medicament. per genera lib. 4, cap. 7 (XIII, 722 K.). Bei Caelius Aurelianus, De morb. acut. II 33, 173 (ed. Drabkin S. 250) und De morb. chron. II 2, 64 (ed. Drabkin S. 606) sind «Aponieus» und «Attaleus» aus Apameus verderbt. Vgl. Caelius Aurelianus, De morb. chron. III 8, 102 (ed. Drabkin S. 776) und V 9, 89 (ed. Drabkin S. 964).

3 Vgl. Plinius, Nat. hist. 26 7, 12. Danach soll der Arzt Asklepiades erst Lehrer der Redekunst in Rom gewesen sein, sich dann aber aus Gewinnstreben der Medizin zugewandt haben.

4 Zum «Abgesandten des Himmels» vgl. Plinius, Nat. hist. 26 7, 13: «universum prope humanum genus circumegit in se non alio modo quam si caelo demissus advenisset.» Die «Totenerweckung» wird u. a. von Celsus, De medicina 2 6, 13/15 (CML I, S. 58 M.) und Plinius, Nat. hist. 7 37, 124 und 26 7, 14/15 überliefert. Apuleius, Florida 4, 19 gibt die ausführlichste Schilderung dieser Begebenheit.

5 Vgl. nur Galen, De natural. facultat. lib. 3, cap. 7 (II, 165 K.), wo vom «lächerlichen Asklepiades» die Rede ist.

${ }^{6}$ Celsus, De medicina 4 9, 2 (CML I, S. 160M.).

7 Vgl. Scribonius Largus, De compos. medicamentorum, praefatio $15 \mathrm{ff}$. (ed. Helmreich S. 3f.) = Marcellus, De medicamentis: Epistula Celsi ad Callistum $7 \mathrm{ff}$. (CML V, ed. Liechtenhan, Bd. 1, S. 38f.). Anlaß der Verteidigung ist das lügnerische Argument, Asklepiades habe behauptet, daß man den Kranken keine Heilmittel geben dürfe. Hierzu bemerkt Scribonius: «Da sich jetzt aber manche in so törichter Weise Schlechtes über ihn ausdenken, was kann ich weiter sagen, als daß diejenigen, die dies behaupten, gewissermaßen Mord und Tempelschändung begehen ... Manche kennen nicht nur die 
alten Autoritäten nicht, durch die der Beruf zu höchster Vollendung gebracht wird, sondern wagen es sogar, sich falsche Behauptungen über sie auszudenken.» Zur Kritik des Scribonius im einzelnen vgl. K. Deichgräber, Professio medici, Abhdl. d. Ak. d. Wiss. und d. Lit. Mainz, Geistes- und Sozialwiss. Kl., Nr. 9, Wiesbaden 1950, S. 862 (10)f.

8 Lukrez, De rerum natura 1, 62-78.

9 Celsus, De medicina 2 6, 13/15 (C.ML I, S. 58 M.).

10 Apuleius, Florida 4, 19: «(Asclepiades) contemplatus etiam atque etiam pertrectavit corpus hominis et invenit in illo vitam latentem.»

11 Das leuchtende mythische Vorbild eines «Totenerweckers» ist natürlich der von Pindar, Pythien 3, $96 \mathrm{ff}$., als Heros apostrophierte Heilgott Asklepios, von dem der späte Nachahmer sogar seinen Namen erhalten hat.

12 Vgl. Daebritz-Funaiole, RE VIII Sp. 472, $32 \mathrm{ff}$., Art. Herakleides Nr. 45. Ferner Herakleides Pontikos in: Die Schule des Aristoteles, Heft VII, hrsg. von F. Wehrli, Basel/ Stuttgart 1969, Texte S. $27 \mathrm{ff}$. und Kommentar S. $86 \mathrm{ff}$.

13 Vgl. besonders Galen, De difficultate respirat. lib. I, cap. 8 (VII, $773 \mathrm{~K}$.), und De locis affectis lib. VI, cap. 5 (VIII, $413 \mathrm{ff}$. K.).

14 Vgl. die Schilderung dieser Begebenheit bei L. Knapp, Der Scheintod der Neugeborenen, Wien/Leipzig 1898, I. Geschichtlicher Teil, S.17f.

15 Genannt seien hier: M. Wellmann, RE II Sp.1632, 8 ff., Art. Asklepiades Nr. 39; H. v. Vilas, Der Arzt und Philosoph Asklepiades von Bithynien, Wien/Leipzig 1903; M. Wellmann, Asklepiades aus Bithynien, von einem herrschenden Vorurteil befreit, Neue Jahrbücher für das Klass. Altertum 21 (1908) 684-703; W.A. Heidel, The anarmoi onkoi of Heraclides and Asclepiades, Transactions of the American Philol. Association 40 (1909) 5-21; Th. Meyer-Steineg, Das medizinische System der Methodiker, Jenaer medizinhistorische Beiträge, Heft 7/8, Jena 1916.

16 Bis heute ist z. B. auch ungeklärt, ob die 1712 unweit der Porta Capena in Rom gefundene Porträtbüste mit der Aufschrift $A \Sigma K \Lambda H \Pi I A \triangle H \Sigma$, die sich jetzt im Kapitolinischen Museum befindet, dem Arzt zuzuweisen ist. Abb. z. B. bei R. H. Major, A history of medicine, Springfield 1954, Bd.1, S.177. Zur Problematik vgl. G. M. A. Richter, The portraits of the Greeks, London 1965, Bd. 3, S. $288 \mathrm{f} .$, Abb. 2055.

17 Die verdienstvolle Fragmentsammlung von Chr. G. Gumpert, Asclepiadis Bithyni fragmenta, praefatus est Chr. G. Gruner, Weimar 1794, ist zusammen mit A. Cocchis Discorso primo sopra Asclepiade, Milano 1748, jetzt in englischer Übersetzung zugänglich bei R. M. Green, Asclepiades, his life and writings, New Haven 1955. Sie ist jedoch unvollständig und trennt den Anteil des Asklepiades Pharmakion nicht immer streng ab.

18 Plinius, Nat. hist. 26 3, 14: «ipse (Asclepiades) cognominari se frigida danda praeferens, ut auctor est M. Varro. » Nicht zuletzt hat aber auch die Nachricht bei Caelius Aurelianus, De morb. acut. I 14, 112 (ed. Drabkin S. 70) der einseitigen Vorstellung vom «Psychrolutes» Vorschub geleistet: «(Asclepiades) laudat etiam in Salutaribus Praeceptis vitae varietatem, atque vehementer utile dicit aquam bibere et frigida lavari, quam psychrolusiam appellant, et frigidam bibere.» Doch hätte nicht nur diese knappe Notiz - sie ist die einzige, die uns Kenntnis von den "Salutaria Praecepta», den Gesundheitsvorschriften, gibt und in diesem Umfang sicher nicht einmal zur Beurteilung dieser Schrift ausreicht - gerade wegen ihres schwer überprüfbaren Aussagewertes vor einer weiteren Verallgemeinerung warnen sollen. Caelius Aurelianus legt vielmehr selbst ein zweifellos 
zutreffenderes Gesamturteil ab, wenn er De morb. acut. II 39, 231 (ed. Drabkin S. 294) schreibt: "hic est ille Asclepiades frigidans atque calefacians, exantlans et replens, et contrariis semper utens rebus in his passionibus.»

19 J. Benedum, Gesnerus 24 (1967) 93-107.

20 Vgl. H. Turner, Sergius Orata, pioneer of radiant heating, Classical Journal 43 (1947/48) 486f. Orata hat nicht die Hypokaustenheizung um 80 v. Chr. erfunden, die auf eine wesentlich längere Entwicklung zurückblickt. Als einfallsreicher Geschäftsmann hat er sie erstmals für Fischzuchtbecken verwandt und später dann auch in Villen einbauen lassen. Arzt und Unternehmer arbeiteten hierbei offenbar eng zusammen.

${ }^{21}$ Vgl. M. Wellmann, RE VI Sp. 333, $26 \mathrm{ff}$., Art. Erasistratos Nr. 2.

${ }^{22}$ Vgl. Gossen-Kind, RE XI Sp. 790, 17 ff., Art. Kleophantos Nr. 2. Sein Werk $\Pi$ ع $i$ olvov $\delta o ́ \sigma \varepsilon \omega_{\varsigma}$ lag nach Celsus, De medicina 3 14, 1 (CML I, S.119M.) der gleichnamigen Schrift des Asklepiades zugrunde. Welchen großen Wert Asklepiades innerhalb seiner Therapie dem Wein beimaß, zeigen die Iatrika des Anonymus Londinensis, XXIV, 30 f. (ed. W. H. S. Jones, S. 94), die den Bithynier als "Oinodótes» charakterisieren. Auch bemerkt Plinius, Nat. hist. 23 22, 38: "Asclepiades utilitatem vini aequari vix deorum potentia posse pronuntiavit.» Zur Weintherapie im Überblick vgl. S. P. Lucia, $A$ history of wine as therapy, Philadelphia 1963, S. $36 \mathrm{ff}$. Daß zahlreiche weitere Ärzte dem Wein eine zentrale Stellung innerhalb der Therapie einräumten, beweisen u. a. auch die Inschriften. So trug z.B. Menekrates aus Tralles, der Sohn des Demetrios, der mit seinem römischen Namen Lucius Manneius Quinti filius Menecrates hieß, ebenfalls den Beinamen eines «Oinodótes». Vgl. IG XIV Nr. $666=$ H. Dessau, Inscr. Lat. Selectae, Berlin 1906, Bd. II, 2, Nr. 7791 = H. Gummerus, Der Ärztestand im römischen Reiche, Helsingfors 1932, Nr. 205. Da seine in Rom gefundene Grabschrift dem 1. Jahrhundert v. Chr. angehört, kann eine direkte Beeinflussung durch Asklepiades nicht ausgeschlossen werden. Schließlich rühmt eine Ehreninschrift des 2.Jahrhunderts n. Chr. aus Athen den Arzt Argaios, weil er Wein als Heilmittel gegen «Gliederschmerzen» verordnete. Vgl. IG III, Nr. $778 / 79$ = G. Kaibel, Epigrammata Graeca, Nr. 853.

${ }^{23}$ Vgl. nur M. Neuburger, Geschichte der Medizin, Bd. 1, Stuttgart 1906, S. 294ff., und Th. Meyer, Theodorus Priscianus und die römische Medizin, Jena 1909, Einleitung S. $5 \mathrm{ff}$.

${ }^{24}$ Vgl. Th. Meyer-Steineg, Das medizinische System der Methodiker, S.16, und Plinius, Nat. hist. 26 8, 14: «iam balneas avidissima hominum cupidine instituendo et alia multa dictu grata atque iucunda ...»

${ }^{25}$ Schon M. Wellmann, Neue Jahrbücher für das Klassische Altertum, S. $688 \mathrm{ff}$., hat die tendenziöse Behauptung des Plinius, Asklepiades habe sich erst in Rom der Medizin zugewandt, als unhaltbar erwiesen.

26 Strabon 12, 566. Angeführt werden u. a. der Grammatiker Asklepiades aus Myrleia und der Arzt Asklepiades.

27 Vgl. bes. Pseudo-Galen, Definitiones cap. 99 (XIX, 373 K.), und Oribasius, Collect. med. XLVII, 12 (CMG VI 2, 1, S. 256 R.), wo beide Male Asklepiades als Bithynier bezeichnet ist.

${ }^{28}$ H. Haeser, Lehrbuch der Geschichte der Medizin, Jena 1875, Bd.1, S. 262. Ausführlich diskutiert H. von Vilas a.a.O. S.12ff. die alte Streitfrage um den Geburtsort des Asklepiades. Vgl. auch Chr. G. Gumpert a.a.0. S.13ff. = R.M.Green a.a.0. S. $57 \mathrm{ff}$.

29 Wir sind Gesnerus 24 (1967) 93-107 dieser communis opinio gefolgt und korrigieren dies hiermit. 
${ }^{30} \mathrm{~L}$. Robert, Sur un type monétaire de Prousa de l'Olympe et sur des épigrammes, Hellenica 2 (1946) 94ff., bes. S. 98, Anm.1. Ferner BCH 1973, Suppl. I, S.437, Anm. 13.

31 Vgl. W. Ruge, RE XI Sp. 486, $30 \mathrm{ff}$., Art. Kios Nr.1.

32 Vgl. F. K. Dörner, RE XXIII Sp. 1128, 16 ff., Art. Prusias ad Hypium Nr. 5. Ferner derselbe, Bericht über eine Reise in Bithynien, Denkschriften der Österr. Ak. d. Wiss., phil.hist. Kl., Bd.75, Abhdl. Nr.1, Wien 1952.

${ }^{33}$ Vgl. F. K. Dörner, RE XXIII Sp.1071, 37 ff., Art. Prusa ad Olympum.

${ }^{34}$ Aus Kios stammte auch jener namentlich unbekannte Mann, der in den epidaurischen Iamata um 320 v. Chr. erwähnt wird. Er wurde im Heiligtum des Gottes von seiner Podagra durch den Biß einer Gans geheilt. Vgl. R. Herzog, Die Wunderheilungen von Epidauros, Philologus Suppl. 22, 3, Leipzig 1931, S. 24ff. Kios selbst scheint nach Ausweis der Münzen erst unter Marc Aurel Asklepios offiziell verehrt zu haben. Vgl. C. Bosch a.a. O. S. $105 \mathrm{f}$.

35 Bezeichnenderweise läßt M. Wellmann, RE II Sp.1632, 8 ff., Art. Asklepiades Nr. 39, die Entscheidung offen, obwohl er zuvor in seiner Pneumatischen Schule, Philolog. Untersuchungen Heft 14, Berlin 1895, S. 6, Anm. 2, das bithynische Prusa als Heimat angegeben hatte.

36 Plinius, Nat. hist. 7 37, 124.

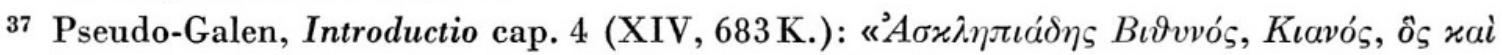

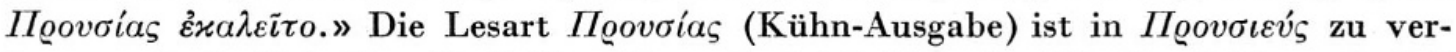

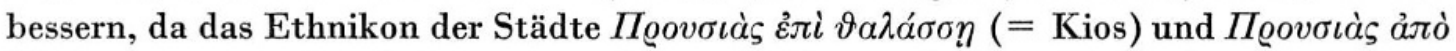

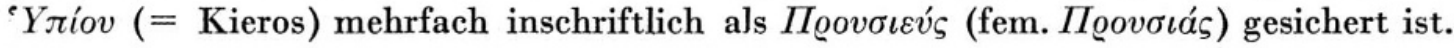
Wenigstens ist nicht anzunehmen, daß Asklepiades als Cognomen den bithynischen

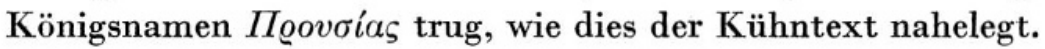

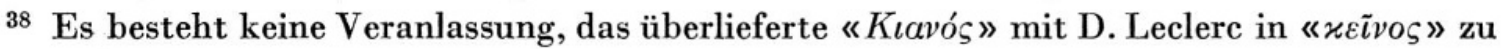
ändern.

${ }^{39}$ Vgl. u. a. E. Babelon/Th. Reinach, Recueil général des monnaies grecques, Bd.1, Fasz. 1, Paris 1904, S. $309 \mathrm{ff}$; Historia Nummorum by B.V. Head, S. $513 \mathrm{f}$. , und Sylloge Nummorum Graecorum Slg. H. v. Aulock, Berlin 1957 ff., Heft 1-3, Nr. 503 ff.; Heft 15, Nr. 7006 (Nachträge).

40 Bildet die claudische Ära (41-54 n. Chr.) die unterste Zeitmarke für die Entstehung der pseudo-galenischen Introductio, so darf die traianische Herrschaft (98-117 n. Chr.) als oberste Grenzmarke gelten. Dies legt nicht nur die Erwähnung des Soran von Ephesos, des Leonidas von Alexandrien und des Archigenes von Apameia in dieser Schrift nahe. Für diesen terminus ante quem spricht vielmehr auch, daß der anonyme Verfasser nur den mutmaßlichen Begründer der methodischen Sekte in Alexandreia Olympikos von Milet (um $100 \mathrm{n}$. Chr.), nicht aber mehr dessen Schulnachfolger Apollonios von Zypern und Julianos zu kennen scheint. Letzterer, den Galen um $155 \mathrm{n}$. Chr. in Alexandreia hörte, lebte noch, als der Pergamener das erste Buch seiner «Therapeutischen Methode» schrieb. Vgl. Galen, De meth. med. lib.1, cap. 7 (X, 53 K.). Kann demnach die Lebenszeit des unbekannten Autors zwischen 40 und $120 \mathrm{n}$. Chr. angesetzt werden, so dürfte auch als Abfassungszeit für die Introductio am ehesten die Jahrhundertwende in Frage kommen.

J. Kollesch, Zur Geschichte des medizinischen Lehrbuchs in der Antike in: Aktuelle Probleme aus der Geschichte der Medizin, Verhandl. des XIX. Internat. Kongr. für 
Geschichte der Medizin, Basel 1966, S. $203 \mathrm{ff} .$, nimmt als Abfassungszeit für die pseudogalenischen Definitiones und die Introductio zu Recht das 1. Jahrhundert n. Chr. an. Vgl.

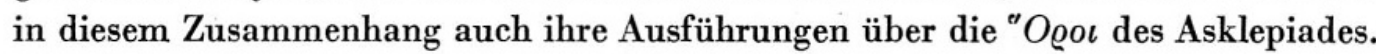

41 Die Frage, inwieweit die pseudo-galenische Introductio aus den Definitiones des Asklepiades entlehnte und noch heute abgrenzbare Teile aufweist, ist beim augenblicklichen Wissensstand nicht zu beantworten. Immerhin hat z. B. auch der Methodiker Mene-

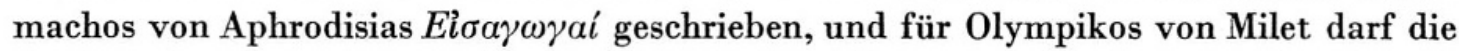
Abfassung von "O@oı angenommen werden. Vgl. Galen, De meth. med. lib. 1, cap. 7 (X, 54 K.). Über die vermutlichen Beziehungen dieser Methodiker zum Anonymus Londinensis vgl. M. Wellmann, Jahrbücher für Klassisches Altertum 21 (1908) 685f., Anm. 5.

${ }^{42}$ Für das alte Kios als Heimat des Asklepiades ist schon H. von Vilas a. a. O. S. $12 \mathrm{ff}$. eingetreten. Zwar muß betont werden, daß diese Nachricht der pseudo-galenischen Introductio durch kein weiteres Zeugnis vorerst gestützt werden kann. Auch ist das ehemalige Kios, das heutige Gemlik, noch kaum erforscht, und die bisher bekannten Inschriften und Münzfunde bieten leider keine weiteren Anhaltspunkte. Doch erweisen sich gerade die geographischen Angaben der Introductio, soweit sie überprüft werden können, als zuverlässig, wenngleich eine kritische Textausgabe auch sonst vermutlich zu einigen unerwarteten Ergebnissen führen könnte. Ähnliche Zuverlässigkeit dürfte auch für die in der Introductio cap. 4 (XIV, $683 \mathrm{~K}$.) angeführten alexandrinischen Chirurgen Apollonios Vater und Sohn aus Antiocheia gelten, die vorerst ohne Grund nicht mit dem bei Soran, Gyn. IV, 14, 3 (CMG IV, S. 144) erwähnten Apollonios von Prusias in Zusammenhang gebracht werden sollten. Vgl. M. Michler a.a.O. S.119ff. Freilich läßt die Angabe

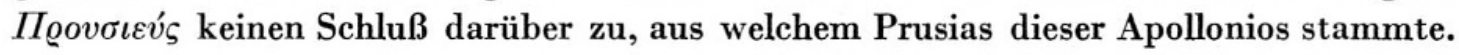

${ }^{43}$ Die Entfernung beträgt rund $30 \mathrm{~km}$. Vgl. die Karte bei A.Philippson, Reisen und Forschungen im westlichen Kleinasien, Heft III (Ergänzungsheft Nr.177 zu «Petermanns Mitteilungen »), Gotha 1913.

44 Oribasius, Collect. med. XLVII, 12 (CMG VI 2, 1, S. 256 R.). Deutsche Übersetzung des Fragments bei H. von Vilas a.a.O. S. 79. Vgl. Chr. G. Gumpert a.a.O. S.181f., § $106=$ R. M. Green a.a.O. S.157, § 106.

45 Caelius Aurelianus, De morb. acut. II 22, 129 (ed. Drabkin S. 218). Vgl. Chr. G. Gumpert a.a.O. S. $102 \mathrm{ff}$., $\S 69=$ R. M. Green a.a.O. S. 110 f., $\S 69$.

46 Vgl. CIL XI Nr. 3943 = R. Briau, L'archiatrie romaine, Paris 1877, S.10ff., Nr.1 = H. Dessau, Inscr. Lat. Selectae, Berlin 1906, Bd. II, 2, Nr. 7789 = H. Gummerus a.a.O. Nr. 242. M. Wellmann, RE II Sp.1633, $51 \mathrm{ff}$., Art Asklepiades Nr. 40, gibt als Geburtsjahr des C. Calpurnius Asclepiades 88 n. Chr. an. Doch nennt die Inschrift das XIII. Konsulat des Domitian, das dem Jahr $87 \mathrm{n}$. Chr. entspricht. Der gelehrte und untadelige Arzt wachte nicht nur im Magistrat der Stadt Rom, sondern auch in den Provinzen, vor allem in der Provincia Asia, bei Prozessen als Kontrolleur (custos) neben der Urne über eine ordnungsgemäße Stimmabgabe. Leider wissen wir nicht, ob er identisch ist mit jenem Asklepiades, der sich als Arzt der Legio II Traiana am 27. Januar $147 \mathrm{n}$. Chr. im ägyptischen Theben eine Inschrift setzte. Vgl. Inscr. Graec. ad res Romanas pertinentes ed. Cagnat, Bd. I, Paris 1911, Nr. 1212. Immerhin wäre vorstellbar, daß der von Traian mit dem römischen Bürgerrecht beschenkte prusische Arzt Asklepiades mit dem Arzt Asklepiades der traianischen Legion in direktem Zusammenhang steht. Denn wir wissen, daß eine Reihe von römischen Zivilärzten zeitweilig im Heer ihren Dienst leistete, zumal 
eine solche Tätigkeit nicht nur hohe Privilegien selbst nach der Rückkehr in den Zivildienst mit sich brachte, sondern auch Gelegenheit bot, an gut ausgestatteten Militärlazaretten große Erfahrungen zu sammeln. Im einzelnen vgl. hierzu die aufschlußreiche Arbeit von R.W. Davies, The medici of the Roman armed forces, Epigraphische Studien Bd. 8, Düsseldorf 1969, S. $83 \mathrm{ff}$.

Die Vermutung W. Wellmanns, C. Calpurnius Asklepiades sei Archiatros Traians gewesen, bleibt ohne Stütze. Auch wird man kaum unter Hinweis auf diesen prusischen Arzt mit F. K. Dörner, RE XXIII Sp.1076, 62 ff., Art. Prusa ad Olympum, folgern können, Prusa sei auch die Geburtsstadt des großen Asklepiades gewesen.

47 Vgl. Corpus Hippiatricorum Graecorum ed. E. Oder/C. Hoppe, Stuttgart 1927, Bd. II, praefatio S. VIf. In Nikomedeia, das ebenfalls als Geburtsort genannt ist, dürfte Apsyrtus zeitweise praktiziert haben, da es als Residenzstadt wohl die größten Möglichkeiten bot. Klazomenai scheint dagegen sein Alterssitz gewesen zu sein. Vgl. hierzu im einzelnen E. Oder, Apsyrtus. Lebensbild des bedeutendsten altgriechischen Veterinärs, Veterinärhistorisches Jahrbuch 2 (1926) $121 \mathrm{ff}$. Zu seiner Lebenszeit vgl. G. Björck, Uppsala Universitets Årsskrift 1944, Nr. 4, S.7-12.

48 Vgl. z.B. die bei F. K. Dörner, RE XXIII Sp.1076, 36ff., Art. Prusa ad Olympum, an-

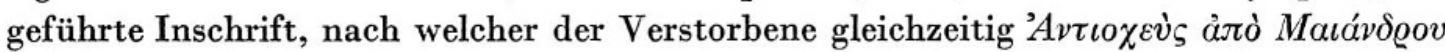

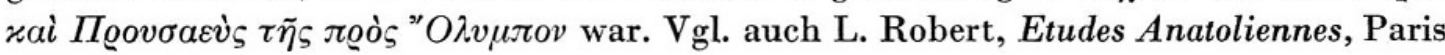
1937, S. 230.

Ferner sei darauf hingewiesen, daß bereits der Großvater und die Eltern des Dio Chrysostomus nicht nur das Bürgerrecht von Prusa von Geburt her besaßen, sondern darüber hinaus auch im Lauf ihres Lebens das apameische und römische Bürgerrecht erwarben. Vgl. H. von Arnim a.a. O. S.123ff.

49 Vgl. J. Platthy, Sources on the earliest greek libraries, Amsterdam 1968, S.166, Nr. 157.

50 Dio Chrysostomus, Orationes 44, 9. Dio hat seine Reden nach der Rückkehr aus seiner Verbannung im Jahre $96 \mathrm{n}$. Chr. gehalten. Er selbst stammte aus einer Familie, welche die höchsten Ämter der Stadt über mehrere Generationen innehatte. Zu seinen bithynischen Reden, die ein anschauliches Bild von den Wirtschaftsverhältnissen und sozialen Einrichtungen der Stadt sowie seinen eigenen Reformbestrebungen entwerfen, vgl. bes. H.von Arnim, Leben und Werke des Dio von Prusa, Berlin 1898. Es mag bezeichnend sein, daß Dio von Prusa in keiner seiner bithynischen Reden des Asklepiades gedenkt, und so hat auch H. von Arnim a.a. O. S.116 klar ausgesprochen, daß Asklepiades nicht aus Prusa, sondern aus dem ehemaligen Kios stammte.

51 Vgl. E. Babelon/Th. Reinach, Recueil général des monnaies grecques, Paris 1912, Bd.1, Fasz. 4, Nr.143; Abb. Taf. CII, 10; F. Imhoof-Blumer, Fluß- und Meeresgötter auf griechischen und römischen Münzen, Revue Suisse de Numismatique 23 (1923), Nr. 231; Abb. Taf. VIII, 6; L. Robert, Sur un type monétaire de Pruse de l'Olympe et sur des épigrammes, Hellenica 2 (1946) 94-102, Abb. Taf. I.

52 Vgl. G. Herzog, RE X Sp. 916 ff., Art. Julia Avita Mamaea Nr. 558.

${ }^{53}$ Zur Frage vgl. G. Fuchs, Architekturdarstellungen auf römischen Münzen der Republik und der frühen Kaiserzeit, Reihe Antike Münzen und geschnittene Steine, hrsg. von E. Boehringer, Berlin 1969.

${ }^{54}$ E. Babelon/Th. Reinach a.a.O. S. 595 erblickten in den beiden Personen Flußgötter. F. Imhoof-Blumer a.a.O. S. 266f. deutete sie jedoch zu Recht als Quellnymphen. 
Vgl. hierzu W. Peek, Griechische Epigramme III, Mitteilungen des Deutschen Archäologischen Institutes Athen 66 (1941) 60 f., Nr. 9. W. Peek bezog versehentlich die Inschrift auf Prusias am Meer. Vgl. dazu L. Robert, Hellenica 2 (1946) 97f., und F. K. Dörner, RE XXIII Sp.1082, $63 \mathrm{ff}$., Art. Prusa ad Olympum.

${ }^{56}$ Die Inschrift lautet nach den Ergänzungen von L. Robert:

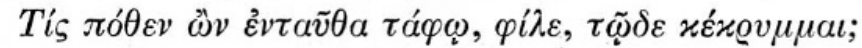

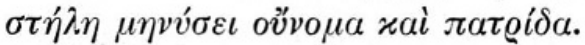

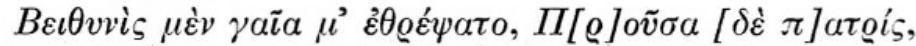

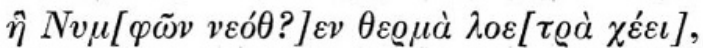

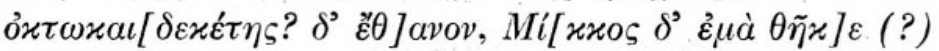

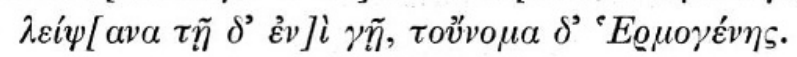

57 Galen, De sanitate tuenda lib. 6, cap. 9 (VI, 424 K.).

58 F. Imhoof-Blumer a.a.O. S. 267.

59 Athenaeus, Deipnosophistae 2 17, 34 (ed. Kaibel, S. 99).

60 Vgl. Plinius, Epistulae 10, 23/24 und 70/71. Näheres bei H. v. Arnim a. a. O. S. 508 f.

61 Vgl. H. von Arnim a.a. O. S. $342 \mathrm{f}$.

${ }^{62}$ Vgl. Plinius, Epistulae 10, 81. In diesem Brief wird auch ein Beamter von Prusa mit Namen Asklepiades erwähnt. Ob dieser mit dem «Wasserdoktor » in Zusammenhang gebracht werden kann, bleibt offen. Ebenso ist vorerst fraglich, ob der in einer Inschrift aus Prusa (2. Jahrhundert n. Chr.) genannte $\tau \varrho \alpha \pi \varepsilon \zeta i \tau \eta \varsigma^{\prime}$ 'A $\approx \varkappa \lambda \eta \pi \iota a ́ \delta \eta \varsigma^{\prime} A \sigma \varkappa \lambda \eta \pi \iota a ́ \delta o v$ mit dem Arzt in verwandtschaftliche Beziehung gesetzt werden kann. Vgl. G. Mendel, Inscriptions de Bithynie, BCH 24 (1900) 366, Nr. 1. Auch sind außer den oben S. 13f. angeführten prusischen Ärzten mit Namen Asklepiades bislang keine weiteren Angehörigen dieses Berufsstandes gleichen Namens aus Prusa bekannt, wenngleich Träger dieses Namens auf Inschriften der Stadt mehrfach begegnen. $\mathrm{Zu}$ einem weiteren prusischen Arzt vgl. F. K. Dörner, Inschriften und Denkmäler aus Bithynien (Istanbuler Forschungen, Bd.14), Berlin 1941, S.112, Nr.131.

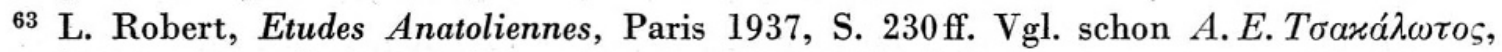

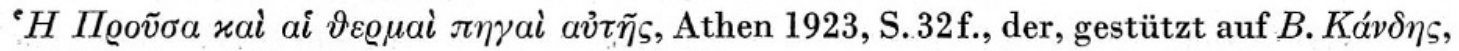

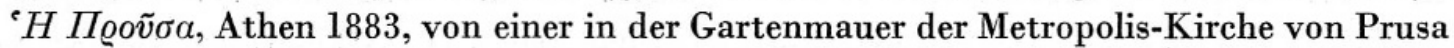

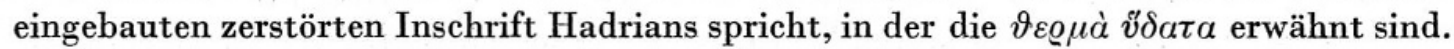
Daß Hadrian sich auch um das öffentliche Bad in Apameia Verdienste erworben hat, beweist eine Inschrift dieser Stadt aus den Jahren 128/129 n. Chr., in der dem Kaiser das «balineum Hadrianum» dediziert wird. Inschrift bei F. K. Dörner, Inschriften und Denkmäler aus Bithynien (Istanbuler Forschungen, Bd.14), Berlin 1941, S.110, Nr.128.

64 Vgl. L. Robert, Hellenica 2 (1946) $98 \mathrm{f}$.

${ }^{65}$ Anthologia Palatina 9, 630 :

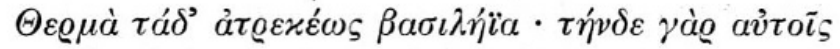

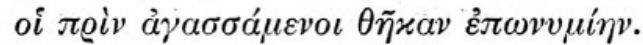

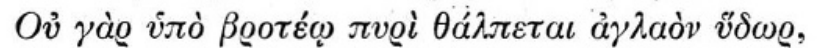
$\alpha \hat{\tau} \tau о \mu \alpha \tau \dot{\eta} \delta \dot{\varepsilon} \lambda_{\iota} \beta \dot{\alpha} \varsigma \tau i \dot{x} \tau \varepsilon \tau \alpha \iota \alpha \dot{\imath} \theta 0 \mu \varepsilon \dot{v} \eta$.

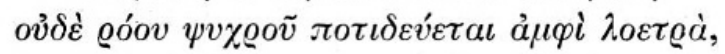

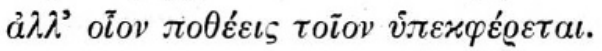


${ }^{66}$ In der Dichtung ist es ein Topos, daß heiße Quellen durch die Liebe eines Gottes erhitzt wurden. In diesem Sinn spricht Ovid (Am. II 6, 23-26) auch die Therma Basilika an. Dazu vgl. R. Merkelbach, ZPE 18 (1975) 178.

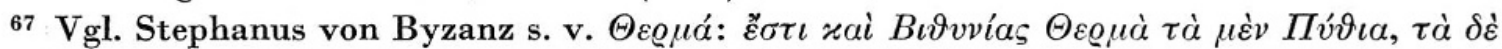

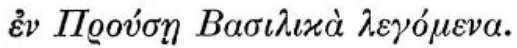

${ }^{68}$ Procopius, De aedificiis 5 3, $16 \mathrm{ff}$.

${ }^{69}$ Anthologia Palatina 9, 676:

$E i \varsigma \pi \eta \gamma \dot{\eta} v \dot{\varepsilon} v{ }^{\prime} O \lambda v \dot{\mu} \mu \omega$ ö $\varrho \varepsilon \iota$.

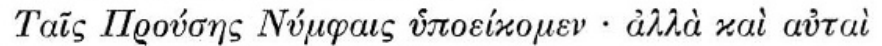

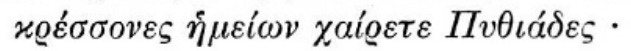

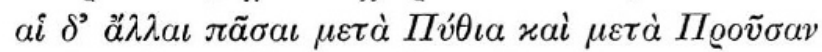

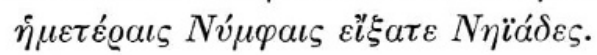

${ }^{70}$ Vgl. J. Hammer, Umblick auf einer Reise von Constantinopel nach Brussa, Pesth 1818, S. 20.

71 Vgl. u. a. A. Grisebach, Reise durch Rumelien und nach Brussa im Jahre 1839, Göttingen 1841, Bd. 1, S. $66 \mathrm{ff}$., und K. v. Fritzsch, Acht Tage in Kleinasien, Mitteilungen des Vereins für Erdkunde zu Halle, Halle 1882, S. $106 \mathrm{ff}$. Weitere Literatur bei H. Inalcık, The Encyclopaedia of Islam, New Edition, Bd. I, Leiden/London 1960, S. $1333 \mathrm{ff}$., Art. Bursa.

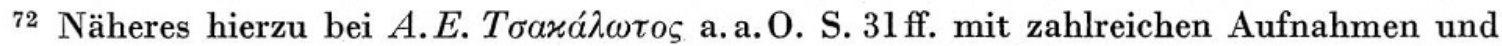

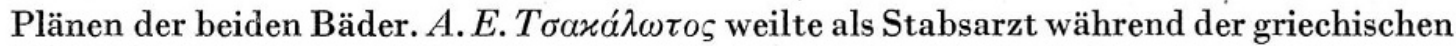
Besatzung längere Zeit in Bursa. Seine Schrift enthält manches Wissenswerte, das man sonst vergeblich sucht. Insbesondere bringt sie eine eingehende Schilderung der berühmten Heilquellen. Vgl. die Besprechung von F. Babinger, Byzantinisch-neugriechische Jahrbücher 5 (1926/27) 472f. Zu Anlage und Architektur dieser Bäder, die wichtige Aufschlüsse über ihre Geschichte geben, vgl. H. Wilde, Brussa. Eine Entwickelungsstätte türkischer Architektur in Kleinasien unter den ersten Osmanen (Beiträge zur Bauwissenschaft Heft 13), Berlin 1909, S. 90 ff.

${ }^{73}$ Zum hamam, insbesondere zum Incirli-hamami und At-Basar-hamami in Brussa vgl. E. Brödner, Heizungstechnische Untersuchungen an islamischen Bädern in Brussa, Bericht der Koldewey-Gesellschaft über die 25.Tagung für Ausgrabungswissenschaft und Bauforschung vom 13.-18. 5. 1969 in Speyer, S. 52-59. Die Verfasserin hebt ebenfalls die Verwandtschaft der Bäder in baulicher und technischer Anlage mit denen der römischen Thermen hervor.

74 Das . Wort kaplica bezeichnet speziell in Bursa eine «natürliche Thermal- und Heilquelle» wie ılıca in der türkischen Sprache überhaupt, im Gegensatz zu hamam, das ein Bad mit künstlich erhitztem Wasser ausdrückt. Aufschlußreich ist darüber hinaus die etymologische Erklärung des Wortes kaphca, das eine «zu einem Bad überdachte Heiß-

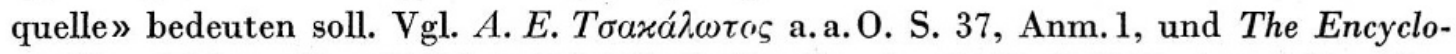
paedia of Islam, New Edition, Bd. III, Leiden/London 1971, S.1120, Art. 1lidja. Man könnte geneigt sein, bereits in dieser sprachlichen Erklärung einen Hinweis auf das mit einem Kuppeldach überwölbte Bad des antiken Münzbildes zu erblicken. Wenigstens stimmt die Bedeutung von Eski Kaplıca als «alte, zu einem Bad überdachte natürliche Heiß- und Heilquelle» auffällig mit den antiken Zeugnissen überein. 
75 Vgl. u.a. A. Philippson a.a.O. S. 71: «Von dem Westende der Stadt sind es nur etwa $2 \mathrm{~km}$ bis zum Dorf Tschekirdje (Çekirge) mit den heißen Quellen Eski Kapludscha (Eski Kaplica) und mehreren Hotels für Badegäste.» Ferner F. K. Dörner, RE XXIII Sp. 1082, $38 \mathrm{ff}$., Art. Prusa ad Olympum. Freilich wird man trotz der großen Übereinstimmung zwischen der antiken und modernen Entfernungsangabe der Tatsache Rechnung tragen müssen, daß die Austrittsstellen der Thermen mehrfach gewechselt haben. Dies zeigen nicht nur die ausgeprägten Sinterbildungen. Bursa ist vielmehr auch häufig von Erdbeben mitgenommen worden, unter denen die heftigsten in der jüngsten Geschichte der Stadt sich im Frühjahr 1855 ereigneten.

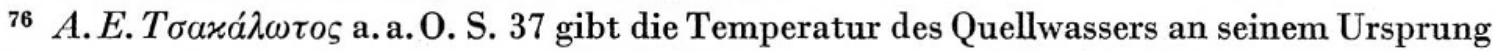
mit $50^{\circ} \mathrm{C}$ an. Nach Fassung und Führung der Quellen durch alte unterirdische Leitungen bis ins Bad und die verschiedenen Becken kühlen sich diese jedoch von selbst bis auf $42^{\circ}$ bis $34^{\circ} \mathrm{C}$ ab.

77 Vgl. F. K. Dörner, RE XXIII Sp.1082, 38 ff., Art.Prusa ad Olympum. In Reclams Kunstführer: Istanbul, Bursa, Edirne, Iznik, Baudenkmäler und Museen; hrsg. v. M. Restle, Stuttgart 1976, S. 471, werden die Pythia nach Çekirge verlegt. Ebenso ist dort von einer «durch nichts zu beweisenden Tradition» des Eski Kaplica die Rede.

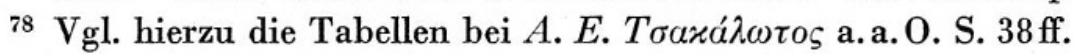

79 A. D. Mordtmann, Anatolien. Skizzen und Reisebriefe aus Kleinasien, Hannover 1925, S. 303.

80 Vgl. schon K. v. Fritzsch a.a. O. S. 108.

81 H.v. Moltke, Briefe über Zustände und Begebenheiten in der Türkei aus den Jahren 1835 bis 1839, 3. Aufl., Berlin 1877, S. 63 ff. Ähnlich begeistert äußert sich A.D.Mordtmann a.a. 0. S. 301 über den Wasserreichtum von Prusa.

Vgl. auch die Schilderung von J. Hammer a.a.O. S. $20 \mathrm{ff}$. Er spricht allein von dreitausend Privatbädern. Freilich dürfte seine Lokalisierung des Eski Kaplıca-Bades im Ostteil der Stadt und nicht im Vorort Çekirge auf einem Irrtum beruhen. Vgl. Taf. III. Zu weiteren Reiseberichten über Brussa vgl. die Zusammenstellung bei J. Sölch, Historisch-geographische Studien über bithynische Siedlungen, Byzantinisch-neugriechische Jahrbücher 1 (1920) 336 f., Anm. 6.

82 CIG II 3283 = Kaibel, EG Nr. 306.

83 Strabo 12, 580. Vgl. J. Benedum, Gesnerus 31 (1974) $221 \mathrm{ff}$.

${ }^{84}$ Für Smyrna als Hauptstätte der von Asklepiades vorbereiteten methodischen Lehre spricht auch der "Arzt und Freund» des Kaisers Augustus Markos Artorios, den literarische Zeugnisse belegen. Vgl. Prosopographia Imperii Romani ed. Groag/Stein I, $236 \mathrm{f}$. Seine in Smyrna gefundene Inschrift (CIG II $3285=$ IGRR IV 1444 = Ehrenberg/Jones, Documents Nr. 143) ist zwar sicher nicht antik. Vgl. schon Sc.Maffei, Mus. Veronense, 1749, S. XLVII, und Pohl, De Graecorum medicis publicis 1905, S. 34. Doch kannte der humanistische Fälscher offenbar seinen Caelius Aurelianus (De morb. acut. III, 14; ed. Drabkin S. 370: Artorius Asclepiadis sectator), wenn er Artorios den Beinamen Asklepiades zulegte. Das inschriftliche Machwerk kann zwar in der erhaltenen Form nicht auf ein antikes Vorbild zurückgehen, doch deutet sein Aufstellungsort Smyrna darauf hin, daß auch der Arzt und Anhänger des Asklepiades M. Artorios mit dieser Stadt in Zusammenhang gebracht werden darf. 


\section{Summary}

During the Greek and Roman antiquity Bithynia has brought forth a number of well-known physicians. One of them is Asclepiades, the pioneer of the Methodist School of medicine and the first important hydrotherapeut of antiquity. A glance at the homeland informs about his doubtful birth-place and shows where Asclepiades received the impulses making him a "water doctor". Here Prusa at Olympus was the most excellent watering-place. Not only the tourists of nowadays travel to these still favoured thermal springs, but in former times Roman rulers, Byzantine emperors and Osman sovereigns also went there to use them. A distance given by Galen and a numismatic testimony furthermore help to identify the antique "Basiliká Thermá» with the modern Turkish Eskikaplıca at Brussa. The further stages of Asclepiades also show that the image of the "psychrolutes" no longer can be maintained.

Prof. Dr. phil. Jost Benedum

Institut für Geschichte der Medizin

Jheringstraße 6

D-63 Gießen 